\title{
COMPLEX-FOLIATED STRUCTURES. I. COHOMOLOGY OF THE DOLBEAULT-KOSTANT COMPLEXES \\ BY
}

\author{
HANS R. FISCHER AND FLOYD L. WILLIAMS
}

\begin{abstract}
We study the cohomology of differential complexes, which we shall call Dolbeault-Kostant complexes, defined by certain integrable subbundles $F$ of the complex tangent bundle of a manifold $M$. When $M$ has a complex or symplectic structure and $F$ is chosen to be the bundle of anti-holomorphic tangent vectors or, respectively, a "polarization" then the corresponding complexes are, respectively, the Dolbeault complex and (under further conditions) a complex introduced by Kostant in the context of geometric quantization. A simple condition on $F$ insures that our complexes are elliptic. Assuming ellipticity and compactness of $M$, for example, one of our key results is a Hirzebruch-Riemann-Roch Theorem.
\end{abstract}

Introduction. The original motivation for the investigation of the "complex-foliated structures" introduced in this paper was provided by the notion of a polarization of a symplectic manifold as defined by Konstant (cf. [10]); we refer to [14] for a concise survey of the developments in the context of geometric quantization. For the cohomological considerations we shall deal with here, the presence of a symplectic form is not required (at least initially) and the important aspects of a polarization now are the "integrability conditions" imposed on a complex subbundle $\mathbf{F}$ of the complexified tangent bundle $T(M)_{\mathrm{C}}$ of $M$. For the sake of brevity we call a subbundle $\mathbf{F} \subset T(M)_{\mathbf{C}}$ which satisfies the "strong integrability conditions" of geometric quantization a complex foliation on $M$ (and apologize for the slight misuse of terminology). Under the conditions of our definition, $F \cap \overline{\mathbf{F}}$ and $\mathbf{F}+\overline{\mathbf{F}}$ induce real foliations $\mathbf{D} \subset \mathbf{E}$ ("involutive" subbundles of $T(M)$ ) and if these are fibrations, one obtains a quotient $M / \mathbf{D}$ with a foliation (fibration) $\mathbf{E} / \mathbf{D}$. In the diagram the bottom map then shows that $M / D$ is a "mixed manifold" in the sense of Kirillov [9] and for this reason, the terminology to be followed had to avoid this term, although it would be well suited to structures $(M, F)$ as will be seen from the basic cohomological results of the present paper.

Received by the editors March 15, 1978 and, in revised form, June 6, 1978.

AMS (MOS) subject classifications (1970). Primary 14F05, 32L10, 58G05, 58G10.

Key words and phrases. Sheaf cohomology, Chern class, Todd class, spectral sequence, elliptic complex. 


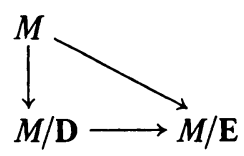

In order to obtain some of the fundamental results, we introduce a direct summand $\mathbf{K}$ of $\mathbf{F}$ in $T(M)_{\mathbf{C}}$, not necessarily integrable, and then the fixed splitting $\mathbf{K} \oplus \mathbf{F}$ of $T(M)_{\mathbf{C}}$ leads to a double grading of the (complex-valued) differential forms on $M$ analogous to the one on a complex manifold or on a real foliated manifold; his latter case was investigated e.g. by Vaisman [20] and to some degree by Sarkaria [17]. We also adapt the splitting of the exterior derivative given in [20] and obtain a decomposition $d=d_{F}+(\partial+\partial)$ with $d_{\mathbf{F}}^{2}=0$ due to the integrability of $\mathbf{F}$. Here $d_{F}$ is of bidegree $(0,1)$. A $p$-form will be called F-holomorphic if it is of type $(p, 0)$ and $d_{\mathrm{F}}$-closed; we choose this terminology in order to emphasize the striking analogy with the complex-analytic case shown in the results of the paper.

The sheaves of forms of type $(p, q)$ and $d_{F}$ now yield a sheaf complex which we shall call the Dolbeault-Kostant complex. This complex is acyclic by the complex Frobenius Theorem due to Nirenberg [12] and therefore yields a fine resolution of the sheaf of F-holomorphic $p$-forms. The result is an analogue of the Dolbeault Theorem in our Theorem 1. For the case $p=0$, this was pointed out by Kostant [10] and a somewhat different proof may also be found in the recent paper [14] by Rawnsley.

In $\S 2$, we introduce F-holomorphic complex vector bundles and extend Theorem 1 to bundle-valued forms and thus obtain the Dolbeault-Serre Theorem of, e.g., [18]. $\$ 3$ is devoted to a special case of interest in geometric quantization: the sheaf complex of a complex line-bundle with a linear connection whose curvature form vanishes on F. Using covariant exterior differentiation, one obtains sheaf complexes analogous to the ones considered earlier and a simple reduction to the scalar case establishes their acyclicity; this leads to a Dolbeault-Serre Theorem for covariant exterior differentiation "along F"; some of the results of $\$ 3$ also can be found in [14]. $\S \S 5$ and 6 present the analogs of two other results well known in complex manifolds: the Frölicher spectral sequence relating the Dolbeault-Kostant and the de Rham cohomologies of $M$, and the Serre Duality Theorem [18], provided that $F$ is elliptic; the latter is obtained following Serre's original arguments without any compactness assumptions on $M$. These results point out a far reaching analogy between our "complex foliations" and complex structures, where F plays a rôle comparable to the one of $T^{0,1}$ in the complex case.

In contrast to what happens in the real case, there are "nontrivial" situations where $d_{F}$ defines an elliptic complex ("F is elliptic"). In fact, a simple necessary and sufficient condition for this is that $\mathbf{F}+\overline{\mathbf{F}}=T(M)_{\mathbf{C}}$, i.e. 
that $\mathbf{E}=T(M)$. If $\mathbf{F}$ is real, this evidently means that $d_{\mathbf{F}}=d$ and, therefore, does not yield any new information. On the other hand, if $\mathbf{F}$ is a polarization of a symplectic manifold, i.e. a maximal totally isotropic (= Lagrangian) subbundle of $T(M)_{C}$, then ellipticitiy of $\mathbf{F}$ implies $\mathbf{F} \cap \bar{F}=0$, hence $T(M)_{C}$ $=\mathbf{F} \oplus \overline{\mathbf{F}}: \mathbf{F}$ is a complex structure and, once again, no new information is obtained. In general, however, the elliptic case leads to some interesting considerations: First of all, the general theory of elliptic complexes now provides finiteness theorems for the cohomologies introduced so far, whenever $M$ is compact. Moreover, the Laplacian $\Delta_{F}$ then is strongly elliptic and therefore its square root $d_{\mathbf{F}}+d_{\mathbf{F}}^{*}$ still is elliptic. This yields the classical elliptic operator $D_{F}$ from forms of types $(0,2 s)$ to the ones of types $(0,2 s+$ 1) and it then is easy to see that the characteristic $\chi_{\mathrm{F}}(M, B)$ of $M$ with coefficients in the sheaf $\delta_{F}(B)$ of holomorphic sections of $\mathbf{B}$ is equal to the analytic index $i_{a}\left(D_{\mathrm{F}}\right)$, hence to the topological index $i_{t}\left(D_{\mathrm{F}}\right)$ by the AtiyahSinger Index Theorem. As a consequence, if we assume $M$ to be compact, even-dimensional and oriented, we can obtain a Riemann-Roch Formula provided that it is possible to find a simple expression for $i_{t}\left(D_{\mathrm{F}}\right)$. Now the symbol of $D_{\mathbf{F}}$ "arises from a universal construction" and $D_{\mathbf{F}}$ is associated with a $G$-structure on $M$ (in the sense of Solovay, [13, Chapter III]) so that the methods of [13] become applicable. The result is the following:

The bundle $(T(M) / D)_{C}=T(M)_{C} / \mathbf{D}_{\mathbf{C}}$ splits into the direct sum $\overline{\mathbf{F}} / \mathbf{D}_{\mathbf{C}} \oplus$ $\mathbf{F} / \mathbf{D}_{\mathbf{C}}$ since $\mathbf{D}_{\mathbf{C}}=\overline{\mathbf{F}} \cap \mathbf{F}$. Therefore, $\overline{\mathbf{F}} / \mathbf{D}_{\mathbf{C}}$ induces a natural complex structure on $T(M) / \mathbf{D}$, the normal bundle of the foliation $\mathbf{D}$. Letting $\mathscr{T}(T(M) / \mathrm{D})$ be the Todd class of this complex vector bundle, $\operatorname{ch}(\mathbf{B})$ the exponential Chern character of the complex vector bundle $\mathbf{B}$ and $e(\mathrm{D})$ the Euler class of the real bundle $\mathbf{D}$, our formula reads:

$$
\chi_{\mathbf{F}}(M, \mathbf{B})=\operatorname{ch}(\mathbf{B}) e(\mathbf{D}) \mathcal{T}(T(M) / \mathbf{D})[M] .
$$

Intuitively, this is an "interpolation formula" between Hirzebruch's Riemann-Roch Theorem $(\mathbf{D}=0)$ and the standard result that the EulerPoincaré characteristic $\chi(M)$ equals $e(T(M))[M](\mathrm{D}=T(M))$. The cohomology class

$$
\mathscr{T}_{\mathbf{F}}(M)=e(\mathbf{D}) \mathcal{T}(T(M) / \mathbf{D})
$$

seems to play a role analogous to that of the Todd class of $M$ in the complex case and provides the interpolation just mentioned. Two assumptions provide the formula: the integrability of $\mathbf{F}$ and the ellipticity of $d_{\mathbf{F}}$; of course, this also applies in the complex case where, however, $\bar{\partial}$ trivially is elliptic, so that the importance of this property generally is less obvious. These matters are presented in $\$ 4$ and applications of the results of this paper will be taken up elsewhere.

Lastly, we should like to mention that the "complex foliations" considered 
here also are related to certain other generalizations of complex structures studied in the literature. The following references give a small (and somewhat arbitrary) sample of the available papers: G. Legrand (Etude d'une généralisation des structures presque complexes, Rend. Circ. Mat. Palermo, 1958), J. Bouzon (Structures presque cocomplexes, Rend. Sem. Mat. Torino, 1964), G. B. Folland and J. J. Kohn (The Neumann problem for the Cauchy-Riemann complex, Ann. of Math. Studies, no. 75, Princeton Univ. Press, Princeton, N. J., 1972), S. S. Chern and Ph. Griffiths (Real hypersurfaces in complex manifolds, Acta Math. 133 (1975)). Details concerning the connections with these structures will appear elsewhere.

0. First of all, we list some of the conventions and notations used throughout this note: The manifolds $M, \ldots$ considered here will be finitedimensional, paracompact $C^{\infty}$-manifolds, unless otherwise specified, and hence will, in particular, admit $C^{\infty}$ partitions of unity. The terms "differentiable" and "smooth" both mean " $C$ " ".

The tangent bundle of $M$ is denoted by $T(M)$, its complexification by $T(M)_{\mathrm{C}}$, the tangent space at $x \in M$ is $T_{x} M$, etc. Also, $T(M)^{*}$ is the real cotangent bundle, $T(M)_{\mathrm{C}}^{*}$ the complex one (= complex dual of $\left.T(M)_{\mathrm{C}}\right)$. The sections of $T(M)_{\mathrm{C}}$ are the complex vector fields on $M$ and the usual bracket $[X, Y]$ extends to these by linearity; this defines a complex Lie algebra structure on the sections of $T(M)_{\mathrm{C}}$. With this, a complex subbundle $\mathbf{F} \subset$ $T(M)_{\mathrm{C}}$ is said to be integrable if the vector space of its sections is a subalgebra of the complex vector fields-by analogy with the real case where this condition amounts to requiring that the subbundle define a foliation of $M$ (by the maximal connected integral manifolds of the bundle). Such a bundle F represents a complex "involutive regular distribution", and conversely. The latter terminology (predating the general use of vector bundles), however, will not be used here. The integrability condition may be re-formulated in terms of the Pfaffian system defining $\mathbf{F}$, exactly as this is done in the real case; moreover, if both $\mathbf{F}$ and $\mathbf{F}+\overline{\mathbf{F}}$ are integrable, $\overline{\mathbf{F}}$ denoting the conjugate of $\mathbf{F}$ with respect to the natural conjugation of $T(M)_{\mathrm{C}}$ over $T(M)$, there is an analogue of the Frobenius Theorem first proved by Nirenberg [12], vaguely justifying our terminology.

If $\mathbf{B}$ is a smooth bundle over $M$ and $U \subset M$ is open, $\Gamma(U, \mathbf{B})$ will denote the set of smooth sections of $\mathbf{B}$ over $U$; if $U=M$, we simply write $\Gamma(\mathbf{B})$ for this set. The $\Gamma(U, \mathbf{B})$ together with the natural restriction maps yield the sheaf $\delta(B)$ of (germs of) smooth sections of B. If $\mathcal{C}$ is the sheaf of smooth complex-valued functions on $M$ and if $\mathbf{B}$ is a vector bundle, then $\delta(\mathbf{B})$ possesses a natural $\mathcal{C}$-module structure which will be understood in the sequel. Also, algebraic operations on vector bundles and the corresponding sheaves of sections will be understood in the appropriate categories. If $\mathscr{F}$ is a 
sheaf over $M$ and $U \subset M$ is open, $\Gamma(U, \mathscr{F})$ denotes the set (group, module,...) of sections of $\mathscr{F}$ over $U$ and again, if $U=M$, we write $\Gamma(\mathscr{F})$ for the set $(\cdots)$ of global sections. Exterior differentiation of (vector-valued) differential forms is denoted by $d$, following standard usage.

Further notational conventions will be introduced as needed.

1. The Dolbeault-Kostant complexes. Let again $M$ be a smooth manifold, $T(M)_{C}$ its complexified tangent bundle.

A complex foliation of $M$ is a complex subbundle $F \subset T(M)_{\mathrm{C}}$ satisfying the following two conditions:

(1) $\mathbf{F} \cap \overline{\mathbf{F}}$ is of constant rank, and both $\mathbf{F}$ and $\mathbf{F}+\overline{\mathbf{F}}$ are integrable.

Since both $\mathbf{F} \cap \overline{\mathbf{F}}$ are stable under conjugation, we immediately see that

$$
\begin{array}{ll}
\mathbf{F}+\overline{\mathbf{F}}=\mathbf{E}_{\mathbf{C}}, & \mathbf{E}=(\mathbf{F}+\overline{\mathbf{F}}) \cap T(M), \\
\mathbf{F} \cap \overline{\mathbf{F}}=\mathbf{D}_{\mathbf{C}}, & \mathbf{D}=(\mathbf{F} \cap \overline{\mathbf{F}}) \cap T(M) .
\end{array}
$$

Moreover, $\mathbf{F}+\overline{\mathbf{F}}$ also is of (locally) constant rank, i.e. a subbundle of $T(M)_{\mathrm{C}}$. Using that $[\bar{X}, \bar{Y}]=\overline{[X, Y]}$ for complex vector fields $X, Y$, we conclude that both $\mathbf{E}$ and $\mathbf{D}$ are integrable and thus define (real) foliations of $M$. We set $n=\operatorname{rank}(\mathbf{F})=\operatorname{rank}(\overline{\mathbf{F}}), k=\operatorname{rank}_{\mathbf{C}}\left(\mathbf{D}_{\mathbf{C}}\right)=\operatorname{rank}(\mathbf{D})$, so that $\operatorname{rank}(\mathbf{E})$ $=2 n-k$.

We obtain a complex "almost product structure" (cf. [20] for the real case) by choosing a direct summand $K$ of $F$ in $T(M)_{C}$, e.g. $K=F^{\perp}$ with respect to some hermitian structure on $T(M)_{\mathbf{C}}$, cf. also below, §4; such a supplement $\mathbf{K}$ is chosen and kept fixed for the remainder of this note. Thus,

$$
T(M)_{\mathbf{C}}=\mathbf{K} \oplus \mathbf{F} .
$$

Once this is done, the splitting $\wedge^{k} T(M)_{\mathbf{C}}^{*}=\bigoplus_{p+q=k}\left(\wedge^{p} \mathbf{K} \otimes \bigwedge^{q} \mathbf{F}\right)^{*}$ leads to a double grading of the sheaf

$$
\Omega=\bigoplus_{k>0} \Omega^{k}
$$

of (germs of) C-valued smooth differential forms on $M$ by setting

$$
\Omega^{k}=\bigoplus_{p+q=k} \Omega^{p, q}
$$

where $\Omega^{p, q}$ is the sheaf of germs of sections of $\left(\bigwedge^{p} \mathbf{K} \otimes \wedge^{0} \mathbf{F}\right)^{*}$. The splitting of $T(M)_{\mathrm{C}}$ also yields a decomposition of [, ]:

$$
[X, Y]=[X, Y]_{1}+[X, Y]_{2}
$$

with $[X, Y]_{1} \in \Gamma(\mathbf{K})$ and $[X, Y]_{2} \in \Gamma(\mathbf{F})$. The integrability of $\mathbf{F}$ means that if $X$ and $Y$ are in $\Gamma(F)$, then so is $[X, Y]$, i.e. $[X, Y]=[X, Y]_{2}$. A slight generalization of the usual splitting of $d$ on a product manifold yields a splitting

$$
d=d_{\mathbf{F}}+d_{1}
$$


where $d_{F}$ is the "exterior derivative along $F$ " and is defined globally by the formula

$$
\begin{aligned}
d_{\mathrm{F}} \beta\left(X_{1}, \ldots, X_{p}, X_{p+1}, \ldots, X_{p+q+1}\right) \\
=(-1)^{p} \sum_{1}^{q+1}(-1)^{i+1} X_{p+i} \beta\left(X_{1}, \ldots, X_{p}, \ldots, \hat{X}_{p+i}, \ldots, X_{p+q+1}\right) \\
\quad+\sum_{i<j}(-1)^{i+j} \beta\left(X_{1}, \ldots, X_{p},\left[X_{p+i}, X_{p+j}\right], \ldots, \hat{X}_{p+i}, \ldots, \hat{X}_{p+j}, \ldots\right) \\
+(-1)^{p} \sum_{i=1}^{p} \sum_{j=1}^{q+1}(-1)^{i+j} \\
\quad \cdot \beta\left(\left[X_{i}, X_{p+j}\right]_{1}, X_{1}, \ldots, \hat{X}_{1}, \ldots, X_{p}, \ldots, \hat{X}_{p+j}, \ldots\right)
\end{aligned}
$$

where $\beta$ is of type $(p, q), X_{i} \in \Gamma(\mathbf{K})$ for $1<i \leqslant p, X_{p+j} \in \Gamma(\mathbf{F})$. The "local formula" is the expected one: If $V \subset H$ is open, $T_{C}(V)=\mathrm{K} \oplus \mathrm{F}$ a splitting of the required type, then

$$
\begin{aligned}
d_{\mathrm{F}} \beta\left(X, \ldots, X_{p+q+1}\right) & \\
= & (-1)^{p} \sum(-1)^{j+1}\left(D \beta \cdot X_{p+j}\right)\left(X, \ldots, \hat{X}_{p+j}, \ldots, X_{p+q+1}\right) .
\end{aligned}
$$

One readily sees that $d_{\mathrm{F}}^{2}=0$ and that $d_{\mathrm{F}}$ is of bidegree $(0,1)$, cf. [20, pp. 184, 185]. The resulting sheaf complex may be called the Dolbeault-Kostant complex. Note that for $p=0$, i.e. for the sheaves $\Omega^{0, q}=\Omega_{\mathrm{F}}^{q}$ of sections of $\left(\wedge^{q} \mathbf{F}\right)^{*}, d_{\mathbf{F}}$ reduces to the standard "exterior derivative along $\mathbf{F}$ "; moreover, these latter sheaves depend only on $\mathbf{F}$, not on the choice of a supplement $\mathbf{K}$ of $\mathrm{F}$ in $T(M)_{\mathrm{C}}$.

In view of later applications, we add a few details on the definitions of $d_{F}$ and of $d_{1}$ :

As usual, a form $\beta$ is of type $(p, q)$ if and only if the following holds: Given the vector fields $X_{1}, \ldots, X_{p+q}$ such that each $X_{i}$ either is a section of $\mathbf{K}$ or a section of $\mathbf{F}$ one has $\beta\left(X_{1}, \ldots, X_{p+q}\right)=0$ except if precisely $p$ of the $X_{i}$ are in $\mathbf{K}$ and $q$ of them are in $\mathbf{F}$, or, a bit more explicitly: suppose that $p^{\prime}$ of the $X_{i}$ are sections of $\mathbf{K}$ and $q^{\prime}$ of them are sections of $\mathbf{F}$. Then $\beta\left(X_{1}, \ldots, X_{p+q}\right)=0$ except when $\left(p^{\prime}, q^{\prime}\right)=(p, q)$, cf. [3].

In the decomposition $\beta=\sum_{p+q=k} \beta^{p, q}$ of an arbitrary $k$-form, the "homogeneous components" $\beta^{p, q}$ are obtained directly by:

$$
\begin{aligned}
\beta^{p, q}\left(X_{1}, \ldots, X_{p+q}\right) & \\
& = \begin{cases}\beta\left(X_{1}, \ldots, X_{p+q}\right) & \text { if } p \text { arguments are in } \mathbf{K}, q \text { are in } \mathbf{F}, \\
0 & \text { otherwise. }\end{cases}
\end{aligned}
$$


Next, assuming that $\beta$ is of type $(p, q)$ and, still, that $\mathbf{F}$ is integrable, write $d \beta=\sum_{r+s=p+q+1} \eta^{r, s}$, where $d$ is the ordinary exterior derivative. Then:

Proposition 1. In $d \beta=\Sigma \eta^{r, s}$, one has $\eta^{r, s}=0$ except for $(r, s)=(p, q+$ $1),(p+1, q)$ or $(p+2, q-1)$.

The result follows as in [20] by examination of the terms

$$
\begin{gathered}
X_{i} \beta\left(X_{1}, \ldots, \hat{X}_{i}, \ldots, X_{p+q+1}\right) \text { and } \\
\beta\left(\left[X_{i}, X_{j}\right], X_{1}, \ldots, \hat{X}_{i}, \ldots, \hat{X}_{j}, \ldots, X_{p+q+1}\right)
\end{gathered}
$$

of $d \beta\left(X_{1}, \ldots, X_{p+q+1}\right)$, a calculation which we do not repeat here.

Note that this result makes use of the integrability condition on $\mathbf{F}$.

Thus, still using the same notations, we have that

$$
d \beta=\eta^{p, q+1}+\eta^{p+1, q}+\eta^{p+2, q-1}
$$

and, clearly, $\eta^{p, q+1}=d_{\mathrm{F}} \beta$. We also define: $\partial \beta=\eta^{p+1, q}, \tilde{\partial} \beta=\eta^{p+2, q-1}$, so that now $d=d_{\mathrm{F}}+\partial+\tilde{\partial}$, i.e. $d_{1}=\partial+\tilde{\partial}$, on forms of a fixed type $(p, q)$. The operators thus defined are extended by linearity to arbitrary forms. As mentioned, one has $d_{\mathbf{F}}^{2}=0$; however, $\partial^{2} \neq 0$, in general, unless $\mathbf{K}$ also is integrable. The following relations which hold in all generality will turn out to be useful later on.

Proposition 2. $\partial^{2}+d_{F} \tilde{\partial}+\tilde{\partial} d_{F}=0, d_{F} \partial+\partial d_{F}=0, \partial \tilde{\partial}+\tilde{\partial} \partial=0, \partial^{2}=0$.

To obtain these, one uses $0=d^{2} \beta$ and $d_{\mathrm{F}}^{2} \beta=0$ and the rearrangement of $d^{2} \beta$ into its homogeneous components, all of which have to vanish. There will be, by the way, the following types: $(p+1, q+1),(p+2, q),(p+3, q-$ 1) and $(p+4, q-2)$.

REMARK. The choice of the symbol $\partial$ as well as some of the later terminology are motivated by the complex-analytic case, where we identify $\mathbf{F}$ with $T(M)^{0,1}$, the antiholomorphic vectors, and $\mathrm{K}$ with $T(M)^{1,0}$.

For each $p \geqslant 0$, there now is a sheaf complex

$$
\Omega^{p \cdot}(\mathbf{C})=\left(\Omega^{p, q}(\mathbf{C}), d_{\mathrm{F}}\right)_{q>0}
$$

on $M$. The sheaf $\operatorname{ker}\left(d_{\mathrm{F}}\right) \subset \Omega^{p, 0}(\mathrm{C})$ is called the sheaf of (germs of) Fholomorphic $p$-forms and will be denoted by $\mathbb{Q}^{p}$. In particular $\mathbb{Q}^{0}=\mathcal{O}_{F}$ is the sheaf of F-holomorphic complex functions on $\boldsymbol{M}$.

Thus, the F-holomorphic $p$-forms are the analogue of the "foliated $p$ forms" considered in [20] for the real case. Moreover, if $k=0$, i.e. $\mathbf{F} \cap \overline{\mathbf{F}}=0$, and $\mathbf{F}+\overline{\mathbf{F}}=T(M)_{\mathrm{C}}$, we are dealing with an integrable almost complex structure on $M$, so that $M$ will be a complex manifold.

With all this, therefore, we have the following sequences:

$$
0 \rightarrow \mathbb{Q}^{p} \stackrel{d_{\mathbf{F}}}{\rightarrow} \Omega^{p, 0}(\mathbf{C}) \stackrel{d_{\mathbf{F}}}{\rightarrow} \Omega^{p, 1}(\mathbf{C}) \stackrel{d_{\mathbf{F}}}{\rightarrow} \cdots \rightarrow \Omega^{p, n}(\mathbf{C}) \rightarrow 0,
$$


$n=\operatorname{rank}(\mathbf{F})$. The sheaves $\Omega^{p, q}(\mathrm{C})$ evidently are fine, being the sheaves of sections of smooth vector bundles. The basic result now is

THEOREM 1. For each $p \geqslant 0$, the sequence (9) is exact: the $\Omega^{p, q}(\mathrm{C})$ yield a fine resolution of $\mathbb{Q}^{p}$ and, therefore,

$$
H^{q}\left(M, \mathbb{Q}^{p}\right)=H^{q}\left(\left(\Gamma\left(\Omega^{p} \cdot(\mathbf{C})\right), d_{\mathbf{F}}\right)\right) .
$$

Proof. The assertion is purely local. Thus, let $M=W$ be an open convex neighbourhood of $0 \in H$ (the parameter space of $M$ ), let $\mathbf{F}$ and $\mathbf{K}$ be trivial subbundles $W \times F, W \times K$ of $W \times H_{\mathrm{C}}$ and $\mathbf{E}=W \times E$. By the Frobenius Theorem, $W$ may be chosen in the form $W=V_{1} \times U$ where $U \subset E$ is a convex neighbourhood of 0 and $V_{1}$ is one of 0 in a summand of $E$.

In addition, using the Nirenberg Theorem [12, Theorem 1'], we conclude that $U=V \times U_{2}$ where $V \subset D$ (= fibre of D), $U_{2} \subset E_{2}, E=D \oplus E_{2}$, in such a way that $E_{2}$ carries a complex structure $J$ with the property that $G=E_{2}^{0,1}$ is a summand of $D_{\mathrm{C}}$ in $F: F=D_{\mathrm{C}} \oplus E_{2}^{0,1}$. Note here, by the way, that the choice of a summand $G \subset F$ of $D_{\mathrm{C}}$ defines such an $E_{2}$ and $J: E_{2}$ is the real part of $G \oplus \bar{G}$ and $J$ is given by: $\langle J\rangle=\left\{(a, b) \in E_{2} \times E_{2} \mid a+i b \in\right.$ $G$.

The last decomposition of $U$ is not invariant under the chart transformations of $M$, but this will not affect the result which concerns $d_{F}$. On $W=V_{1} \times V \times U_{2}$, we treat the $a \in V_{1}$ as "parameters" in the formulas to be established since differentiation in these directions will not enter the arguments directly.

Let now $p \geqslant 0$ be fixed. The local product structure $U=V \times U_{2}$ leads to a splitting of $d_{F}$ and a double grading of $\Omega^{p, q}(C)$ in $q$ : we have $\left(\bigwedge^{q} F\right)^{*}=$ $\bigoplus_{r+s=q}\left(\bigwedge^{r} D_{\mathbf{C}} \otimes \bigwedge^{s} G\right)^{*} ; \Omega^{p, q}(\mathrm{C})$ is the sheaf of germs of smooth maps $W \rightarrow\left(\bigwedge^{p} K \otimes \bigwedge^{q} F\right)^{*}$, whence the double grading with respect to $q$ and, consequently, the splitting of $d_{F}$ into $d_{1}+d_{2}$ with $d_{1}^{2}=0$ and $d_{2}^{2}=0$ (cf. [6] or [20]).

We obtain a double complex $\bar{K}=\left(K^{r s}, d_{1}, d_{2}\right)$ by taking for $K^{r s}$ the stalk at 0 of the sheaf $\Omega^{p, r, s}$ and for $d_{1}, d_{2}$ the two differentials just defined.

The first claim is that $H_{I}^{r}(\bar{K})=0$ for $r>1$ :

Define a "partial Poincaré homotopy" $k$ by

$$
\begin{aligned}
& k \beta(a, x, y)\left(u_{1}, \ldots, u_{p}, x_{1}, \ldots, x_{r-1}, y_{1}, \ldots, y_{s}\right) \\
& \quad=(-1)^{p} \int_{0}^{1} t^{r-1} \beta(a, t x, y)\left(u_{1}, \ldots, u_{p}, x, x_{1}, \ldots, x_{r-1}, y_{1}, \ldots, y_{s}\right) d t .
\end{aligned}
$$

The standard calculation shows immediately that $k d_{1}+d_{1} k=\mathrm{id}$ (cf. also [20, p. 215]). Finally, since $\beta$ is smooth in $(a, x, y)$, so is $k \beta$ and the claim follows. 
Moreover, we also have $H_{\mathrm{II}}^{s}(\bar{K})=0$ for $s \geqslant 1$ :

Using an argument of Cartan and Weil (cf. [21, p. 71] and [3, p. 97]), we see that there is a neighbourhood $U_{0} \subset U_{2}$ of 0 such that if $\beta$, defined on $V_{1} \times V \times U_{2}$, satisfies $d_{2} \beta=0$ there, then $\beta \mid V_{1} \times V \times U_{0}$ is real-analytic in $y$. Writing $z$ for the "complex coordinates" defined in $U_{0}$ by $J, \beta$ extends to $\beta(a, x, z, \bar{z})$ in the usual manner, the coefficients of the power series then being smooth in $(a, x)$. In this case, we set

$$
\begin{aligned}
& K \beta(a, x, z)\left(u_{1}, \ldots, u_{p}, x_{1}, \ldots, x_{r}, y_{1}, \ldots, y_{s-1}\right) \\
& =(-1)^{p+r} \int_{0}^{1} t^{s-1} \beta(a, x, z, t \bar{z})\left(u_{1}, \ldots, x_{r}, \bar{z}, y_{1}, \ldots, y_{s-1}\right) d t,
\end{aligned}
$$

a "partial Dolbeault homotopy". One concludes $K d_{2}+d_{2} K=$ id on realanalytic forms and the assertion follows.

A simple spectral sequence argument now completes the proof: $H^{q}(\bar{K})=0$ for $q \geq 1$.

REMARK. The auxiliary double grading in $q$ makes sense globally if we select a summand $\mathbf{G}$ of $\mathbf{D}_{\mathbf{C}}$ in $\mathbf{F}$. If $\mathbf{G}$ is integrable, then the local "mixed structure" globalizes to some extent to the leaves of the foliation given by $\mathbf{E}$. In fact, $\mathbf{F}$ already defines a generalized "mixed manifold structure" (cf. [9] for the terminology). In the presence of further restrictions on $\mathbf{D}$ and $\mathbf{E}$ ("strongly admissible F"), more can be said about the connection with the concepts introduced in [9], cf. [15], [10]; cf. also \$4 concerning G.

If $B$ is a complex vector space, then the same arguments will still hold for the sheaves $\Omega^{p, q}(B)$ of $B$-valued forms:

COROLlaRY 1. The cohomology of $M$ with coefficients in the sheaf $\mathbb{Q}^{p}(B)$ of $B$-valued F-holomorphic p-forms is given by

$$
H^{q}\left(M, \mathbb{Q}^{p}(B)\right)=H^{q}\left(\left(\Gamma\left(\Omega^{p, q}(B)\right), d_{\mathrm{F}}\right)\right) .
$$

CoRollary 2. If $q>n=\operatorname{rank}(\mathbf{F})$, then $H^{q}\left(M, \mathbb{Q}^{p}(B)\right)=0$.

We refer also to [14] for another proof of Theorem 1.

2. The analogue of the Dolbeault-Serre Theorem. We now consider a further generalization of Theorem 1: Let $\mathbf{B}$ be a complex vector bundle over $M$ with fibre $B$. We say that $\mathbf{B}$ is $\mathbf{F}$-holomorphic if there exists a vector bundle atlas $\left(U_{\alpha}\right)$ on $M$ such that the 1-cocycle $\left(g_{\alpha \beta}\right)$ representing B for this atlas satisfies

$$
d_{\mathbf{F}} g_{\alpha \beta}=0
$$

meaning that each $g_{\alpha \beta}$ is an F-holomorphic map $U_{\alpha} \cap U_{\beta}=U_{\alpha \beta} \rightarrow \mathrm{GL}(B) \subset$ $L(B)$.

If $\mathcal{G}, \mathcal{G}_{\mathbf{F}}$ are the sheaves of smooth and F-holomorphic maps, resp., of $\boldsymbol{M}$ into $\mathrm{GL}(B)$, then B will be F-holomorphic iff its isomorphism class lies in the 
image of $H^{1}\left(M, \mathcal{G}_{\mathrm{F}}\right) \rightarrow H^{1}(M, \mathcal{G})$, the map being induced by the inclusion $\mathcal{S}_{\mathbf{F}} \rightarrow \mathcal{G}$.

Let $b \in \Gamma(\mathbf{B})$ be represented by the maps $b_{\alpha}: U_{\alpha} \rightarrow B$ in the given trivializations $\mathbf{B} \mid U_{\alpha} \simeq U_{\alpha} \times B$. Then, over $U_{\alpha \beta}$,

$$
b_{\beta}=g_{\beta \alpha} b_{\alpha}
$$

Using the fact that $d_{F}$ on (vector-valued) maps simply is the exterior derivative restricted to $\mathbf{F}$, we have that $d_{\mathrm{F}} b_{\beta}=\left(d_{\mathrm{F}} g_{\beta \alpha}\right) b_{\alpha}+g_{\beta \alpha} d_{\mathrm{F}} b_{\alpha}=g_{\beta \alpha} d_{\mathrm{F}} b_{\alpha}$ because of (12). Thus, the maps $d_{\mathbf{F}} b_{\alpha}$ represent a global section of $\mathbf{B}$ and this section will be denoted by $d_{\mathrm{F}} b$. As before, the operator $d_{\mathrm{F}}$ defined on $\mathbf{B}$ now leads to corresponding operators on the sheaves $\Omega^{p, q}(\mathbf{B})$ of $\mathbf{B}$-valued forms of type $(p, q)$; this yields the sheaf complexes $\left(\Omega^{p, q}(\mathrm{~B}), d_{\mathrm{F}}\right)_{q>0}$. Evidently, the sheaves $\Omega^{p, q}(B)$ still are fine and we now conclude from Corollary 1 of Theorem 1, denoting by $\mathbb{Q}^{p}(B)$ the sheaf of F-holomorphic B-valued $p$-forms:

THEOREM 2. For each $p \geqslant 0,\left(\Omega^{p}(\mathbf{B}), d_{\mathrm{F}}\right)$ is a fine resolution of $\mathbb{Q}^{p}(\mathbf{B})$ and therefore

$$
H^{q}\left(M, \mathbb{Q}^{p}(\mathbf{B})\right)=H^{q}\left(\left(\Gamma\left(\Omega^{p \cdot}(\mathbf{B})\right), d_{\mathrm{F}}\right)\right) .
$$

In particular, $H^{q}\left(M, \mathbb{Q}^{p}(\mathbf{B})\right)=0$ for $q>n=\operatorname{rank}(\mathbf{F})$.

This is the analogue of the Serre-Dolbeault Theorem (which holds for holomorphic vector bundles on a complex manifold [18]). Questions of possible analogues of Serre's duality theorems will be dealt with later. At this point, note that if $\mathbf{B}$ is $\mathbf{F}$-holomorphic, then so is its dual $\mathbf{B}^{*}$ : transposition is a linear map $L(B) \rightarrow L\left(B^{*}\right)$ and, therefore, $d^{t} g={ }^{t} d g$ for any map $g$ into $L(B)$; if $d_{\mathrm{F}} g=d g \mid \mathbf{F}$ vanishes, so does $d_{\mathrm{F}}{ }^{t} g$. The analyticity of the inversion in $\mathrm{GL}(B)$ or $\mathrm{GL}\left(B^{*}\right)$ is not needed here because of $g_{\alpha \beta}^{-1}=g_{\beta \alpha}$.

In fact, one readily argues that the class of F-holomorphic vector bundles over $M$ is closed under ${ }^{*}, \otimes$ and $\oplus$.

REMARK. Let $\mathcal{C}$ be the sheaf of germs of smooth complex functions on $M$ and denote by $\theta_{F}$ the sheaf of germs of F-holomorphic functions. This is a subsheaf (of local rings) of $C$ so that any $C$-module also is an $\hat{\theta}_{\mathbf{F}}$-module. Moreover, the sheaves $Q^{p}(\mathbf{B})$ introduced here are $\Theta_{\mathbf{F}}$-modules (but not, in general, $\mathcal{C}$-modules) since for a section $b$ of $\mathbf{B}$ and a complex function $f$, one clearly has $d_{\mathrm{F}}(f b)=\left(d_{\mathrm{F}} f\right) b+f d_{\mathrm{F}} b$. This also shows that if $b$ is a local section without zeroes, then $f b$ is holomorphic ( $b$ being holomorphic) if and only if $f$ is. There follows the usual observation: the sheaf $\mathbb{Q}^{0}(\mathbf{B})=\mathcal{S}_{\mathbf{F}}(\mathbf{B})$ of $\mathbf{F}$ holomorphic sections of $\mathbf{B}$ is locally free over $\theta_{F}$ of local rank at each point equal to rank (B), and the $\Gamma\left(\mathcal{O}_{\mathbf{F}}\right)$-module $\Gamma\left(\delta_{\mathbf{F}}(\mathbf{B})\right)=\Gamma_{\mathbf{F}}(\mathbf{B})$ of F-holomorphic sections of $B$ is a finitely generated projective over $\Gamma\left(\Theta_{F}\right)$. One has the standard category equivalences and, in particular, the theory of Fholomorphic vector bundles of finite rank is equivalent with the theory of 
locally free $\hat{\theta}_{\mathbf{F}}$-modules (of finite rank, of course). Here, the F-holomorphic line bundles correspond to the invertible $\hat{\theta}_{\mathbf{F}}$-modules and, as always, their isomorphism classes form an abelian group $\mathscr{P}_{\mathbf{F}}(M)$ under $\otimes$, isomorphic to $H^{1}\left(M, \theta_{F}^{*}\right), \theta_{F}^{*}$ the sheaf of germs of nonvanishing $\mathbf{F}$-holomorphic functions.

3. The complex of a line-bundle with connection. We add the following remarks on complex line-bundles over $M$ in view of their geometric interest (geometric quantization, representation theory, cf. [10], [15], etc.):

If the complex line-bundle $\mathbf{L}$ is $\mathbf{F}$-holomorphic, then all the preceding considerations, of course, apply. We write, as before, $\mathcal{S}_{\mathbf{F}}(\mathrm{L})$ for the sheaf of F-holomorphic sections of $\mathbf{L}$ and also $\Omega_{\mathbf{F}}^{q}(\mathbf{L})$ for $\Omega^{0, q}(\mathbf{L})$. In addition, we shall use the following notations: $\mathrm{C}^{*}$ is the group of nonzero complex numbers and $\mathbf{L}^{+}$is the principal bundle associated with $\mathbf{L}$ (the frame bundle of $\mathbf{L}$ ), identified in the usual way with the complement of the zero-section in $\mathbf{L}$. If $\boldsymbol{\nabla}$ is a linear connection on $L, \omega$ its connection form, then the curvature form $\Omega$ simply is $d \omega$ (the group $\mathbf{C}^{*}$ being abelian) and is an integral 2-form on $\boldsymbol{M}$.

The connection $\nabla$ may now be used to define a differential operator $\nabla_{F}$, the "covariant exterior derivative along $\mathbf{F}$ ", firstly for sections of $\mathbf{L}$ and then for L-valued differential forms, by replacing the directional derivatives in the first term of the right-hand side of (6) by covariant derivatives. In general, $\nabla_{\mathrm{F}}^{2} \neq 0$; in fact, $\nabla_{\mathrm{F}}^{2} \beta=\Omega(\beta)$ (in the notations e.g. of $[6, \mathrm{II}] ; \Omega \wedge \beta$ in the notations of [2]).

We say that $\nabla$ and $\mathbf{F}$ are compatible if $\Omega$ vanishes on $\mathbf{F}$, i.e. if $\mathbf{F}$ is totally isotropic for $\Omega$. The formula $\nabla_{F}^{2} \beta=\Omega(\beta)$ implies that if $\nabla$ and $F$ are compatible, then

$$
\nabla_{\mathbf{F}}^{2}=0
$$

Thus, we now also obtain the sheaf complexes $\left(\Omega^{p} \cdot(L), \nabla_{\mathrm{F}}\right), p>0$, whose acyclicity will be established below by a "reduction to the scalar case" for which we use the following proposition, of interest in itself (the last assertion was conjectured and used, e.g., in [10], etc.):

Proposition 3. The following statements are equivalent:

(a) $\mathbf{L}^{+}$admits local sections $s$ with $\nabla_{\mathbf{F}} s=0$.

(b) Every point of $M$ has a neighbourhood on which $\Omega=d \alpha$ for some 1-form $\alpha$ satisfying $\alpha \mid \mathbf{F}=0$.

Moreover, if $\nabla$ and $\mathbf{F}$ are compatible, then (a) and thus (b) hold true.

Proof. Let $s$ be any local section of $\mathbf{L}^{+}$, i.e. a local nonvanishing section of L. We define a 1-form $\alpha_{s}$ by

$$
\nabla_{X} s=\alpha_{s}(X) s,
$$

$X$ a vector field. One easily sees that $d \alpha_{s}=\Omega$ on the domain of $\alpha_{s}$ : one has 
$\left(\nabla_{X} \nabla_{Y}-\nabla_{Y} \nabla_{X}\right) s=\left(X \alpha_{s}(Y)-Y \alpha_{s}(X)\right) s$ from the definition $\alpha_{s}$, and the claim follows.

The implication (a) $\Rightarrow(\mathrm{b})$ is now clear: $\nabla_{X} s=0$ for the sections of $X$ of $F$ obviously implies $\alpha_{s} \mid \mathbf{F}=0$. Conversely, suppose that there is a 1-form $\alpha$ with $\alpha \mid \mathbf{F}=0$ and $\Omega=d \alpha$ on some open set. Then $d\left(\alpha-\alpha_{s}\right)=0$ and, on a sufficiently small open set, $\alpha=\alpha_{s}+d g$ for some function $g$. Set $s_{0}=e^{g_{s}}$. Then $s_{0}$ has no zero and we obtain that $\nabla_{X} s_{0}=\nabla_{X}\left(e^{g_{s}}\right)=\left(d g+\alpha_{s}\right)(X) e^{z_{S}}=$ $\alpha(X) s_{0}$ which vanishes if $X$ is a section of $F$, so that $\nabla_{F} s_{0}=0$.

To establish the second assertion, let $s$ be local section of $\mathbf{L}^{+}$. Then $t=f s$ satisfies $\nabla_{\mathbf{F}} t=0$ if and only if the function $f$ satisfies

$$
d_{\mathrm{F}} f=-f \alpha_{1}
$$

where $\alpha_{1}$ is (the extension by 0 of) $\alpha_{s} \mid F$; therefore, it suffices to find a nonvanishing solution of this equation. On a sufficiently small open set, the nonvanishing $f$ will be of the form $f=e^{g}$, in which case $d f=e^{g} d g$. Thus, we have to find a local solution of

$$
d_{\mathrm{F}} g=-\alpha_{1} .
$$

To do this, observe that $\alpha_{1}$ is of type $(0,1)$ so that $d_{F} \alpha_{1}(X, Y)=X \alpha_{1}(Y)-$ $Y \alpha_{1}(X)-\alpha_{1}([X, Y])=X \alpha_{s}(Y)-Y \alpha_{s}(X)-\alpha_{s}([X, Y])=d \alpha_{s}(X, Y)=$ $\Omega(X, Y)=0$ for any two sections $X, Y$ of F. By Theorem 1, there is indeed a function $g$ with $d_{\mathrm{F}} g=-\alpha_{1}$ and we are done.

REMARK. Observe that if $(\mathbf{L}, \nabla)$ satisfies (a), then $\mathbf{L}$ is F-holomorphic: let $t$ be any local section of $\mathrm{L}^{+}, s$ one with $\nabla_{\mathrm{F}} s=0$. We may write $t=f s$ for some nonvanishing function $f$. But then $\nabla_{\mathrm{F}} t=0$ if and only if $d_{\mathrm{F}} f=0$. Therefore, if (a) holds near any given point of $M$, then the transition functions for the local trivializations obtained in this manner will be F-holomorphic maps into $C^{*}$.

With these remarks, we obtain the

THEOREM 3. For each $p \geqslant 0$, the complex $\left(\Omega^{p, q}(\mathbf{L}), \nabla_{\mathrm{F}}\right)_{q>0}$ is acyclic and hence yields a fine resolution of the sheaf $\mathbb{Q}_{\nabla}^{p}(\mathrm{~L})$ of $\mathbf{L}$-valued forms of type $(p, 0)$ satisfying $\nabla_{\mathbf{F}} \gamma=0$ (“covariant F-holomorphic p-forms"):

$$
H^{q}\left(M, \mathbb{Q}_{\nabla}^{p}(\mathbf{L})\right)=H^{q}\left(\left(\Gamma\left(\Omega^{p} \cdot(\mathbf{L})\right), \nabla_{\mathrm{F}}\right)\right) .
$$

In particular, if $\mathcal{S}_{\mathbf{F}}^{\nabla}(\mathbf{L})$ is the sheaf $\mathscr{Q}_{\nabla}^{0}(\mathbf{L})$ sections of $\mathbf{L}$ satisfying $\nabla_{\mathbf{F}} s=0$, then

$$
H^{q}\left(M, \mathcal{S}_{\mathbf{F}}^{\nabla}(\mathbf{L})\right)=H^{q}\left(\left(\Gamma\left(\Omega_{\mathbf{F}}(\mathbf{L})\right), \nabla_{\mathbf{F}}\right)\right) \text {. }
$$

Proof. Let $\beta \in \Gamma\left(U, \Omega^{p, q}(\mathbf{L})\right)$ and $s \in \Gamma\left(U, \mathbf{L}^{+}\right)$with $\nabla_{F} s=0$. Write $\beta=\hat{\beta} \cdot s$ where now $\hat{\beta}$ is a scalar form of type $(p, q)$. Then $\nabla_{\mathrm{F}} \beta=\nabla_{\mathrm{F}}(\hat{\beta} \cdot s)$ $=\left(d_{F} \hat{\beta}\right) s+\hat{\beta} \cdot \nabla_{F} s$; the second term vanishes and we see that $\nabla_{F} \beta=0$ if and only if $d_{F} \hat{\beta}=0$. With this, the claim is reduced to the scalar case dealt with in Theorem 1. 
REMARKS. (1) The last assertions apply, in particular, to the case of a polarized symplectic manifold $(M, \Omega)$ and a complex line bundle $\mathbf{L}$ with a linear connection whose curvature form is $\Omega$. In this case, $F$ will be a maximal totally $\Omega$-isotropic subbundle of $T(M)_{\mathrm{C}}$, cf. the literature (e.g. [10], [15], . . ). We also refer to [14] for very similar results, in particular to Theorem 3 of this reference.

(2) The generalization of the last considerations to complex vector bundles of rank $>1$ does not seem to be clear at this time; we shall return to this problem later.

4. A Riemann-Roch Theorem for elliptic foliations. Letting $L(A, B)$ denote the bundle of linear maps $\mathbf{A} \rightarrow \mathbf{B}$, it is evident that $\Omega^{p, q}(\mathbf{B})$ is the sheaf of sections of $L\left(\bigwedge^{p} \mathbf{K} \otimes \bigwedge^{q} \mathbf{F}, \mathbf{B}\right)=\mathbf{D}^{p, q}(\mathbf{B})$. If $\mathbf{B}$ is $\mathbf{F}$-holomorphic, we thus have a sequence

$$
\mathbf{D}^{p, 0}(\mathbf{B}) \stackrel{d_{\mathbf{F}}}{\rightarrow} \mathbf{D}^{p, 1}(\mathbf{B}) \stackrel{d_{\mathbf{F}}}{\rightarrow} \cdots \rightarrow \mathbf{D}^{p, n}(\mathbf{B}) \rightarrow 0
$$

of bundle maps. $d_{\mathbf{F}}$ is a first order differential operator (at each $\left.p, q\right)$ and we now wish to determine its symbol:

Let $\xi \in T_{x}^{*} M$ (real cotangent space!), $\beta \in \mathbf{D}^{p, q}(\mathbf{B})_{x}$. The symbol $\sigma\left(d_{F}\right)$ is calculated in the usual way: Choose a function $f$ near $x$ such that $d f(x)=\xi$ and let $s$ be a section of $D^{p, q}(B)$ with $s(x)=\beta$. Then

$$
\sigma\left(d_{\mathbf{F}}\right)(x)(\xi, \beta)=\left(d_{\mathbf{F}}(f-f(x)) s\right)(x) \text {. }
$$

The result is the following: Denote by $\xi_{F}$ the restriction to $F_{x}$ of the complex extension of $\xi$. Then

$$
\sigma\left(d_{F}\right)(x)(\xi, \beta)=(-1)^{p}\left(\xi_{F} \wedge \beta\right) .
$$

One concludes immediately that the symbol sequence is exact for $\xi \neq 0$ if and only if the following condition is satisfied:

$\left(\mathrm{E}_{1}\right)$ If $\xi \neq 0$ in $T_{x}^{*} M$, then $\xi_{F} \neq 0$.

This condition may be translated into a somewhat simpler one by means of the following brief argument from linear algebra: Given the real vector space $H$ and a complex subspace $F$ of $H_{\mathrm{C}}$, we claim that condition $\left(\mathrm{E}_{1}\right)$ for $\xi \in H^{*}$, $\xi \neq 0$, is equivalent with $F+\bar{F}=H_{\mathbf{C}}$ : Indeed, if this condition is satisfied and if $\xi_{F}=0$, then also $\xi_{\bar{F}}=0$ by the reality of $\xi$, therefore $\xi=0$. Conversely, suppose that $F+\bar{F} \subsetneq H_{\mathrm{C}}$ and let $E \subset H$ be the real part of $F+\bar{F}$, i.e. $E=(F+\bar{F}) \cap H$. Then $E \subsetneq H$ and therefore there exists $\xi \in H^{*}, \xi \neq 0$ with $\xi \mid E=0$. But then $\xi_{F}=0$, contradicting $\left(\mathrm{E}_{1}\right)$.

This yields 
THEOREM 4. The condition

(E) $\mathbf{F}+\overline{\mathbf{F}}=T(M)_{\mathbf{C}}$

is necessary and sufficient for the following to hold:

(1) Given the F-holomorphic vector bundle B, for each $p>0$ the complex $\left(\mathbf{D}^{p, q}(\mathbf{B}), d_{\mathrm{F}}\right)_{q>0}$ is elliptic.

(2) For $p \geq 0$ and the line bundle $\mathbf{L}$ with a compatible linear connection $\nabla$, the complex $\left(\mathbf{D}^{p, q}(\mathbf{L}), \nabla_{\mathbf{F}}\right)_{q>0}$ is elliptic.

COROllary. If $M$ is compact and if $\mathbf{F}$ satisfies (E), then the cohomologies $H^{\bullet}\left(M, \mathbb{Q}^{p}(\mathrm{~B})\right), H^{\bullet}\left(M, \mathbb{Q}_{\nabla}^{p}(\mathrm{~L})\right)$ are finite-dimensional.

The proof of (2) is as straightforward as the one of (1) since formula (14) merely uses the fact that $d_{\mathbf{F}}$ is a derivation, and this also holds for $\nabla_{\mathbf{F}}$.

RemarKs. (1) Assume that $\beta \in \mathbf{D}^{p, q}(\mathbf{B})_{x}$ is decomposable: $\beta=\gamma \wedge \delta$ with $\gamma \in \mathbf{D}^{p, 0}(\mathbf{B})_{x}$ and $\delta \in \mathbf{D}^{0, q}(\mathbf{B})_{x}$. Then $(-1)^{p}\left(\xi_{F} \wedge \beta\right)=\gamma \wedge\left(\xi_{F} \wedge \delta\right)$. In this sense, $\sigma\left(d_{F}\right)$ is obtained by "tensoring" the symbol on $D^{0, q}(\mathbf{B})=L\left(\wedge^{q} \mathbf{F}, \mathbf{B}\right)$ with the identity and thus is the standard extension of the symbol of $d_{F}$ defined on the "pure F-forms".

(2) Theorem 4 shows that in the case of a polarized symplectic manifold, ellipticity of the complexes involved is possible only if $\mathbf{F} \oplus \overline{\mathbf{F}}=T(M)_{\mathrm{C}}$, i.e. if $M$ is a Kähler manifold. Thus, the approach does not lead to any new information in this special case.

(3) Using $\mathbf{E}=(\mathbf{F}+\overline{\mathbf{F}}) \cap T(M)$ again, condition (E) clearly is equivalent with:

$\left(\mathrm{E}^{*}\right) \mathbf{E}=T(M)$.

REMARK. If one assumes that the foliation defined by $\mathbf{D}$ is a fibre bundle $M \rightarrow M / D$, then one can argue that $M / D$ will be a complex manifold. This will not be pursued here; however, cf. below for a discussion of an approach leading to such cases.

If one chooses hermitian structures on the bundles $\mathbf{D}^{p, q}(\mathbf{B})$, one can define the (formal) adjoint $d_{\mathbf{F}}^{*}$ of $d_{\mathbf{F}}$ and, consequently, a Laplacian operator $\Delta_{\mathbf{F}}$. The latter will be an elliptic operator of order 2 whose symbol is given by

$$
\sigma\left(\Delta_{\mathbf{F}}\right)=\sigma\left(d_{\mathbf{F}}^{*}\right) \sigma\left(d_{\mathbf{F}}\right)+\sigma\left(d_{\mathbf{F}}\right) \sigma\left(d_{\mathbf{F}}^{*}\right)=-\left(\sigma\left(d_{\mathbf{F}}\right)^{*} \sigma\left(d_{\mathbf{F}}\right)+\sigma\left(d_{\mathbf{F}}\right) \sigma\left(d_{\mathbf{F}}\right)^{*}\right),
$$

which will be negative-definite (cf. below, also [13, p. 73]).

Letting $\mathcal{F}_{\mathbf{F}}^{p, q}(\mathbf{B})$ be the space of $\Delta_{\mathbf{F}}$-harmonic sections of $D^{p, q}(B)$, the orthogonal projection onto the harmonic sections induces an isomorphism

$$
H^{q}\left(\left(\Gamma\left(\Omega^{p} \cdot(\mathbf{B})\right), d_{\mathbf{F}}\right)\right) \cong \mathcal{F}_{\mathbf{F}}^{p, q}(\mathbf{B})
$$

whence the corollary to Theorem 4 in the usual manner.

One observes that these results are somewhat more precise than the ones obtained in [20] for the real case. In fact, the only real case subsumed under 
the current considerations is the one where $\mathbf{F}=\mathbf{D}_{\mathbf{C}}$ (so that $\overline{\mathbf{F}}=\mathbf{F}$ ), so that ellipticity amounts to $\mathbf{D}=T(M)$ !

In order to make the sequel independent of the (arbitrary) choice of a summand $\mathbf{K}$ of $\mathbf{F}$ in $T(M)_{\mathbf{C}}$, we limit ourselves to the case of forms of type $(0, q)$. Again, the sheaf $\mathbb{Q}^{0}(\mathbf{B})$ of F-holomorphic sections of the F-holomorphic complex vector bundle $\mathbf{B}$ is denoted by $\delta_{\mathbf{F}}(\mathbf{B})$.

The main assumptions we make here are: $M$ is closed and oriented, and $\mathbf{F}$ is elliptic (i.e. satisfies condition (E) of §4).

We know that under these conditions, the (generalized) Hodge theorem guarantees that the cohomology groups which appear in the current considerations all are finite-dimensional. In particular, it makes sense to define the Euler-Poincaré characteristic.

$$
\chi_{\mathbf{F}}(M, \mathbf{B})=\chi\left(M, \delta_{\mathbf{F}}(\mathbf{B})\right)=\sum(-1)^{q} \operatorname{dim}\left(\mathcal{F}_{\mathbf{F}}^{0, q}(\mathbf{B})\right)
$$

in the usual manner, cf. e.g. [8], [13], [22], etc. Note that if $B \equiv M \times C=1$ is the trivial line-bundle (which is, of course, F-holomorphic!), then $\chi_{F}(M, 1)$ generalizes the arithmetic genus (following the conventions adopted in [8]) of a compact complex manifold to our situation.

In view of our previous results, we intend to obtain a Riemann-Roch Theorem through an application of the index theorem for elliptic differential operators to a suitably constructed operator; the construction parallels the classical argument (cf. e.g. [13, Chapter XIX]): We choose a Riemannian structure on $M$ and extend it to a hermitian structure on $T(M)_{C}, F, F^{*}$ and their exterior powers in the usual manner, and we fix a hermitian structure on B. Then, the bundles

$$
\mathbf{D}^{0, q}(\mathbf{B})=L\left(\bigwedge^{q} \mathbf{F}, \mathbf{B}\right)=\bigwedge^{q} \mathbf{F}^{*} \otimes \mathbf{B}
$$

carry hermitian structures which will be the ones we use henceforth. The operator $d_{\mathrm{F}}: \mathbf{D}^{0, q}(\mathbf{B}) \rightarrow \mathrm{D}^{0, q+1}(\mathrm{~B})$ now has an adjoint $d_{\mathrm{F}}^{*}$ defined using the given hermitian structures and the same holds for $d_{F}$ on $\Gamma\left(D^{0, q}(B)\right)$ (where we also use the given Riemann measure on $M$ ); from the general theory of elliptic complexes, we conclude that the Laplacian $\Delta_{\mathrm{F}}=d_{\mathrm{F}} d_{\mathrm{F}}^{*}+d_{\mathrm{F}}^{*} d_{\mathrm{F}}=$ $\left(d_{\mathbf{F}}+d_{\mathbf{F}}^{*}\right)^{2}$ has the property that

$$
\Delta_{\mathrm{F}} \omega=0 \text { if and only if } d_{\mathrm{F}} \omega=0 \text { and } d_{\mathrm{F}}^{*} \omega=0 \text {, }
$$

whence also: $\Delta_{\mathbf{F}} \omega=0$ if and only if $\left(d_{\mathbf{F}}+d_{\mathbf{F}}^{*}\right) \omega=0$.

Set now

$$
\begin{aligned}
\mathbf{D}^{+}(\mathbf{B}) & =\bigoplus \mathbf{D}^{0,2 k}(\mathbf{B}), \quad \mathbf{D}^{-}(\mathbf{B})=\bigoplus \mathbf{D}^{0,2 k+1}(\mathbf{B}), \\
D_{\mathbf{F}} & =d_{\mathbf{F}}+d_{\mathbf{F}}^{*}: \Gamma\left(\mathbf{D}^{+}(\mathbf{B})\right) \rightarrow \Gamma\left(\mathbf{D}^{-}(\mathbf{B})\right) .
\end{aligned}
$$

Then, evidently $D_{\mathbf{F}}^{*}=d_{\mathbf{F}}^{*}+d_{\mathbf{F}}: \Gamma\left(\mathbf{D}^{-}(\mathbf{B})\right) \rightarrow \Gamma\left(\mathbf{D}^{+}(\mathbf{B})\right)$. The first claim is 
Proposition 4. $D_{\mathbf{F}}$ is elliptic.

For the proof, observe that $D_{\mathbf{F}}^{*} D_{\mathrm{F}}=\Delta_{\mathrm{F}}$, so that it is sufficient to show that $\Delta_{F}$ is strongly elliptic [13, p. 73], i.e. that $-\sigma\left(\Delta_{F}\right)$ is positive definite as a function of $\xi \in T^{*}(M)$ (real cotangent bundle!). Now, denoting by $\phi_{\xi}$ the map $\omega \rightarrow \xi_{F} \wedge \omega$ used earlier and by $i_{\xi}$ its adjoint (in the given hermitian structures), formula (14) shows that $\sigma\left(\Delta_{F}\right)(x)(\xi, \beta)=\left(i_{\xi} \varphi_{\xi}+\phi_{\xi} i_{\xi}\right) \beta$ and the usual computation (cf. e.g. $\left[13\right.$, p. 77]) yields $\sigma\left(\Delta_{F}\right)(\xi, \beta)=-\left\|\xi_{F}\right\|^{2} \cdot \beta$. Since $F$ is elliptic, the assertion is proved.

Now $\operatorname{ker}\left(D_{\mathrm{F}}\right)$ consists of the harmonic forms of type $(0,2 k)$ and $\operatorname{ker}\left(D_{\mathrm{F}}^{*}\right)$ of the ones of type $(0,2 k+1)$. Thus, the definition of $\chi_{\mathbf{F}}(M, \mathbf{B})$ immediately implies

Proposition 5. $\chi_{\mathbf{F}}(M, \mathbf{B})=i_{a}\left(D_{\mathbf{F}}\right), i_{a}$ denoting the analytic index of the operator in question (notations of [13]). It is now clear that a Riemann-Roch Theorem will be obtained once the topological index $i_{t}\left(D_{\mathrm{F}}\right)$ can be brought into a suitable form.

The reduction will be done following the ideas and methods of [13, Chapter III] (Solovay): we wish to interpret $D_{F}$ as an elliptic differential operator associated with a $\mathcal{G}$-structure on $M, \mathcal{G}$ a compact Lie group. In order to determine a natural such group $\mathcal{G}$ and actions on the fibres of the bundles involved, we firstly have to add some linear algebra, taking into account the "mixed" structures we are dealing with:

Let $E$ be the fibre of $T(M), D$ the one of D, F the one of F. Then $E_{\mathrm{C}}=F+\bar{F}$ and $D_{\mathrm{C}}=F \cap \bar{F}$ by our assumptions. Here, the bar denotes the natural conjugation in $E_{\mathrm{C}}$ over $E$. Let $E$ be oriented and choose a euclidean structure $($,$) on E$. This extends in a standard way to a hermitian structure on $E_{\mathbf{C}}$, still denoted by $($,$) . Observe that \overline{(g, h)}=(\bar{g}, \bar{h})$ for this hermitian structure.

Next, let $E_{0}=D^{\perp} \subset E$, so that $E=D \oplus E_{0}$ is an orthogonal splitting. Also, let $G=D_{\mathbf{C}}^{\perp} \cap F$ in $E_{\mathbf{C}}$, so that $F=D_{\mathbf{C}} \oplus G$; similarly, $\bar{F}=D_{\mathbf{C}} \oplus \bar{G}$. Set $\operatorname{dim}(E)=2 l$, the odd-dimensional case being of little interest here. If $n=\operatorname{dim}_{\mathrm{C}}(F)=\operatorname{dim}_{\mathrm{C}}(\bar{F})$ and $k=\operatorname{dim}(D)$, then $2 n-k=2 l$ and so $k=$ $2 d, n=l+d$; moreover, $\operatorname{dim}_{\mathrm{C}}(G)=\operatorname{dim}_{\mathrm{C}}(\bar{G})=l-d$.

Since $D$ is oriented, it is an $S O(2 d)$-module. Similarly, $G$ becomes a $U(l-d)$-module in a natural way. In addition, there now is a natural action of $U(l-d)$ on $E_{0}$ which we obtain as follows:

$G$ defines an almost-complex structure $J$ on $E_{0}$ by: $y=J x$ iff $x+i y \in G$, i.e. $G$ is the "graph" of $J$. In this case, extending $J$ by linearity to $E_{0, c}$, we obtain the projections $\frac{1}{2}(1+i J): E_{0, \mathrm{C}} \rightarrow G, \frac{1}{2}(1-i J): E_{0, \mathrm{C}} \rightarrow \bar{G}$, etc., so that $G=E_{0}^{0,1}$ and $\bar{G}=E_{0}^{1,0}$ with respect to $J \in G L\left(E_{0}, \mathbf{R}\right)$. Moreover, $U(l-d)$ acts on $\bar{G}$ by essentially the contragredient action, using that 
$\bar{G} \cong G^{*}$ under the hermitian structure of $E_{\mathbf{C}}$. Explicitly, this means that for $a \in U(l-d)$ and $h \in \bar{G}$, we set: $a h=\overline{a \bar{h}}$. If $x \in E_{0}$, set $g=\frac{1}{2}(1+i J) x$, $\bar{g}=\frac{1}{2}(1-i J) x$. Then $x=g+\bar{g}$ is the unique decomposition of $x$ in $E_{0, \mathrm{C}}=$ $G \oplus \bar{G}$. We define

$$
a x=a g+\overline{a g}, \quad a \in U(l-d) .
$$

Clearly, this defines an $R$-linear operation and, moreover, $a J=J a$ on $E_{0}$ : firstly, $a(1+i J)=a+i a J$, of course. Thus, if $g=\frac{1}{2}(1+i J) x$, then $a g$ $=\frac{1}{2}(a x+i a J x)$. Thus, $a x$ is the real part of $a g$ and therefore $a g=\frac{1}{2}(1+$ $i J) a x$-from which it follows immediately that $a J=J a$ on $E_{0}$. As a consequence, the action of $U(l-d)$ on $G \oplus \bar{G}$, defined by $a(g+h)=a g$ $+a \bar{h}$, is the complexification of the action on $E_{0}$. In other words: $E_{0, \mathrm{C}}$ is the complexified $U(l-d)$-module of $E_{0}$. Summarizing, we see that $E=D \oplus E_{0}$ is an $S O(2 d) \times U(l-d)$-module whose complexification is $D_{\mathrm{C}}+E_{0, \mathrm{C}}=$ $E_{\mathrm{C}}, S O(2 d)$ acting on $D_{\mathrm{C}}$ by extension.

It is important for our purposes to observe that these module structures are defined "globally" on $M$, i.e. on the corresponding bundles: $M$ is oriented and the choice of a Riemannian structure on $M$ leads to the constructions just outlined on each fibre of $T(M)=\mathbf{E}, T(M)_{\mathbf{c}}=\mathbf{E}_{\mathbf{C}}=\mathbf{F}+\overline{\mathbf{F}}, \mathbf{D}_{\mathbf{C}}=\mathbf{F} \cap \overline{\mathbf{F}}$, $\mathbf{E}_{0}=\mathbf{D}^{\perp} \subset T(M), \mathbf{G}=\mathbf{D}_{\mathbf{C}}^{\perp} \cap \mathbf{F}$. In particular, $\mathbf{E}_{0}$ carries an almost-complex structure induced by $\mathbf{G}$ and the bundles in question admit as their structural groups $S O(2 d), U(l-d)$ and-for $T(M)=\mathbf{E}$ as well as $\mathbf{F}=\mathbf{D}_{\mathbf{C}} \oplus \mathbf{G}, \overline{\mathbf{F}}=$ $\mathbf{D}_{\mathbf{C}} \oplus \overline{\mathbf{G}}-\boldsymbol{S} O(2 d) \times U(l-d)$.

Also choose a hermitian structure on the bundle $\mathbf{B}$ whose fibre is, say, a complex vector space $B$ of dimension $b$, so that B becomes a $U(b)$-bundle. Set

$$
\mathcal{G}=S O(2 d) \times U(l-d) \times U(b)
$$

and let this group act on the various fibres by: $\mathcal{G} \rightarrow S O(2 d) \times U(l-d)$, the latter acting on $\mathbf{E}, \mathbf{F}$ as before, and $\mathcal{G} \rightarrow U(b)$ for the action on $\mathbf{B}$. Then, if $P$ is the principal $\mathcal{G}$-bundle associated with $T(M)$, we have: $T(M)=P$ $\times_{g} E, \mathbf{F}=P \times_{\mathcal{G}} F, \mathbf{G}=P \times_{\mathcal{G}} G, \mathbf{B}=P \times_{\mathcal{G}} B$, etc.

For the computation of the weights of the various actions we also need the following observation: We may change the euclidean structure on $E_{0}$ to $(x, y)_{1}=(x, y)+(J x, J y)$, on $E$ to $(x, y)+(\tilde{J} x, \tilde{J} y)$ where $\tilde{J}$ is the endomorphism $0+J$ of $E=D \oplus E_{0}$; then, still, $E_{0}=D^{\perp}, G \perp D_{\mathrm{C}}$, $\bar{G} \perp D_{\mathrm{C}}$, etc., for the corresponding hermitian structure on $E_{\mathbf{C}}$ (where now also $G \perp \bar{G}$ !) and now $J$, restricted to $E_{0}$, is orthogonal and skew-symmetric. In particular, if $x \in E_{0}$, then $x \perp J x$ and the two vectors have the same length.

Now, given $x \in E_{0}$, let $\rho x=\frac{1}{2}(1-i J) x \in \bar{G}$. The plane $[x, J x]$ has an obvious complex structure: $\alpha+\beta i$ operates as $\alpha x+J \beta x$ and this complex 
line is isomorphic to $C \cdot \rho x$ since $\alpha x+J \beta x$ maps to $(\alpha+\beta i) \rho x$, as a short verification shows. Given $\beta \in \mathbf{R}$, denote by $\theta_{\beta}$ the automorphism of $[x, J x]$ (where $\|x\|=\|J x\|=1$ ) given by the $2 \times 2$-matrix

$$
\left(\begin{array}{cc}
\cos 2 \pi \beta & -\sin 2 \pi \beta \\
\sin 2 \pi \beta & \cos 2 \pi \beta
\end{array}\right)
$$

in the basis $\{x, J x\}$. If $h \in \bar{G}$, then $h$ easily is normalized so that for $x=\rho^{-1} h,\{x, J x\}$ is an orthonormal basis of the plane $[x, J x]$. Next, choose a basis $e_{1}, \ldots, e_{l-d}$ of $\bar{G}$ such that a given maximal torus $T \subset U(l-d)$ operates by

$$
t e_{j}=\exp \left(2 \pi i \beta_{j}(t)\right) e_{j} ;
$$

the $\beta_{j}$ are the weights of the $U(l-d)$-module $\bar{G}$ (also, by definition, of the $\mathcal{G}$-module $\bar{G}$ ). Setting $x_{j}=\rho^{-1} e_{j}, T$ leaves $\left[e_{j}, J e_{j}\right]$ invariant and operates on this plane by $\theta_{\beta_{j}}$; in fact, for any $\beta$,

$$
e^{2 \pi i \beta} \rho x=\rho\left(\theta_{\beta} x\right) .
$$

This implies that the weights of $E_{0}$ are $\beta_{j}, i \leqslant j \leqslant l-d$. Thus, the weights of $E_{0}$ and $\bar{G}$ agree and those of $G$ are $-\beta_{j}$.

The euclidean structure $(,)_{1}$ yields an isomorphism of $E$ onto $E^{*}$ (as $\mathcal{G}$-modules); letting $\alpha_{1}, \ldots, \alpha_{d}$ be the weights of $D$, we see from [13, p. 35 and pp. 41, 42] that the Euler class $e\left(T(M)^{*}\right)$ is $\Pi \alpha_{j} \cdot \Pi \beta_{j}$. Next, the weights of $F^{*} \cong \bar{F}$ are $\pm \alpha_{j}, \beta_{j}$. Since the symbol of $D_{\mathrm{F}}$ is induced by a "universal construction", namely the map $\xi \rightarrow \phi_{\xi}-\phi_{\xi}^{*}$ of the sphere bundle of $T^{*}(M)$ into the isomorphisms $\oplus \wedge^{2 s} \mathbf{F}^{*} \otimes \mathbf{B} \rightarrow \bigoplus \wedge^{2 s+1} \mathbf{F}^{*} \otimes \mathbf{B}$, the "Chern character" of $D_{\mathbf{F}}$ is computed by

$$
\operatorname{ch}\left(D_{\mathbf{F}}\right)=\operatorname{ch}(\mathbf{B}) \Pi \frac{\left(1-e^{\alpha_{j}}\right)\left(1-e^{-\alpha_{j}}\right)}{-\alpha_{j}} \Pi \frac{1-e^{\beta_{j}}}{-\beta_{j}}
$$

following [13, pp. 36 and 40]; the signs in the denominator yield the factor $(-1)^{l}$ needed in the definition of $\operatorname{ch}\left(D_{\mathbf{F}}\right)$, cf. [13, p. 29].

Next, the Todd classes of the complex bundles $\mathbf{D}_{\mathbf{C}}, \mathbf{G}$ and $\overline{\mathbf{G}}$ are obtained as follows:

$$
\begin{aligned}
\mathscr{T}\left(\mathbf{D}_{\mathbf{C}}\right) & =\Pi \frac{\alpha_{j}}{1-e^{-\alpha_{j}}} \cdot \frac{-\alpha_{j}}{1-e^{\alpha_{j}}}, \\
\mathcal{T}(\mathbf{G}) & =\Pi \frac{-\beta_{j}}{1-e^{\beta_{j}}}, \\
\mathcal{T}(\overline{\mathbf{G}}) & =\Pi \frac{\beta_{j}}{1-e^{-\beta_{j}}} ;
\end{aligned}
$$

since $\mathscr{T}\left(T(M)_{\mathbf{C}}\right)=\mathscr{T}\left(\mathbf{D}_{\mathbf{C}}\right) \mathcal{T}(\mathbf{G}) \mathscr{T}(\overline{\mathbf{G}})$ from $T(M)_{\mathbf{C}}=\mathbf{D}_{\mathbf{C}} \oplus \mathbf{G} \oplus \overline{\mathbf{G}}$, we see that $i_{t}\left(D_{\mathrm{F}}\right)$ is represented by the cohomology class 


$$
\begin{aligned}
\operatorname{ch}\left(D_{\mathbf{F}}\right) \mathscr{T}\left(T(M)_{\mathbf{C}}\right)= & \operatorname{ch}(\mathbf{B}) \prod \frac{\left(1-e^{\alpha_{j}}\right)\left(1-e^{-\alpha_{j}}\right)}{-\alpha_{j}} \Pi \frac{1-e^{\beta_{j}}}{-\beta_{j}} \\
& \cdot \prod \frac{\alpha_{j}}{1-e^{-\alpha_{j}}} \frac{-\alpha_{j}}{1-e^{\alpha_{j}}} \prod \frac{-\beta_{j}}{1-e^{\beta_{j}}} \prod \frac{\beta_{j}}{1-e^{-\beta_{j}}} \\
= & \operatorname{ch}(\mathbf{B}) \prod \alpha_{j} \mathcal{T}(\overline{\mathbf{G}}) ;
\end{aligned}
$$

observing now that the remaining middle term is $e(\mathrm{D})$ (real $S O(2 d)$-bundle!), we are done; the Atiyah-Singer Theorem implies the following formula:

$$
\chi_{\mathbf{F}}(M, \mathbf{B})=\operatorname{ch}(\mathbf{B}) e(\mathbf{D}) \mathcal{T}(\bar{G})[M] .
$$

Next, since $\mathbf{E}_{\mathbf{0}}$ is assumed to carry the complex structure $J$ defined by $\mathbf{G}$, it is immediate that $\mathbf{E}_{\mathbf{0}} \cong \overline{\mathbf{G}}$ over $\mathbf{C}$, so that the Todd class on the right-hand side also is $\mathscr{T}\left(\mathbf{E}_{0}\right)$; moreover, $T(M)=\mathbf{D} \oplus \mathbf{E}_{0}$ where now $\mathbf{E}_{0}$ is a complex subbundle determined by $\mathbf{F}$ and the Riemannian structure chosen earlier. This last choice may be eliminated in the following way: Clearly, $\mathbf{E}_{0} \cong$ $T(M) / \mathrm{D}$ under the natural map in the exact sequence $0 \rightarrow \mathrm{D} \rightarrow T(M) \rightarrow$ $T(M) / \mathbf{D} \rightarrow 0$. The complexification of this map yields an isomorphism of $\mathbf{E}_{0, \mathbf{C}}=\mathbf{G} \oplus \overline{\mathbf{G}}$ onto $T(M)_{\mathbf{C}} / \mathbf{D}_{\mathbf{C}}=\mathbf{F} / \mathbf{D}_{\mathbf{C}} \oplus \overline{\mathbf{F}} / \mathbf{D}_{\mathbf{C}}$ which maps $\mathbf{G}$ to $\mathbf{F} / \mathbf{D}_{\mathbf{C}}$ and $\overline{\mathbf{G}}$ to $\overline{\mathbf{F}} / \mathbf{D}_{\mathbf{C}}$. Since evidently $T(M)_{\mathbf{C}} / \mathbf{D}_{\mathbf{C}}=(T(M) / \mathbf{D})_{\mathrm{C}}$, the splitting of this bundle into $\mathbf{F} / \mathbf{D}_{\mathbf{C}} \oplus \overline{\mathbf{F}} / \mathbf{D}_{\mathbf{C}}$ defines a complex structure $J_{\mathbf{D}}$ on $T(M) / \mathbf{D}$ and it is immediate from the construction that $\left(E_{0}, J\right) \cong\left(T(M) / D, J_{\mathbf{D}}\right)$, i.e. that $\mathrm{E}_{0}$ and $T(M) / \mathrm{D}$ are isomorphic as complex vector bundles under the natural map $T(M) \rightarrow T(M) / D$.

With this, we now obtain

THEOREM 5 (RIEMANN-ROCH FORMULA). Let $M$ be closed, even-dimensional and oriented, F elliptic. If $T(M) / \mathbf{D}$ carries the complex structure $J_{\mathbf{D}}$ induced by $\mathbf{F}$, then for any $\mathbf{F}$-holomorphic vector bundle $\mathbf{B}$ over $M$,

$$
\chi_{\mathbf{F}}(M, \mathbf{B})=\operatorname{ch}(\mathbf{B}) e(\mathbf{D}) \mathcal{T}(T(M) / \mathbf{D})[M] .
$$

REMARK. The class $e(D) \mathcal{T}(T(M) / D)$ apparently plays the role of "generalized Todd class of $M$ " and may be denoted by $\mathscr{T}_{\mathbf{F}}(M)$; in this case, (24) reads as follows:

$$
\chi_{\mathbf{F}}(M, \mathbf{B})=\operatorname{ch}(\mathbf{B}) \mathscr{T}_{\mathbf{F}}(M)[M] .
$$

In the two classical special cases, one gets back known theorems: Suppose, firstly, the $\mathbf{F}$ is real, i.e. that $\mathbf{F}=\mathbf{D}_{\mathbf{C}}=\mathbf{E}_{\mathrm{C}}=T(M)_{\mathrm{C}}$, and that $\mathbf{B}=1$ is the trivial line bundle. Then $d_{\mathbf{F}}=d, T(M) / \mathbf{D}=0, \delta_{\mathbf{F}}(1)=\mathbf{C}$ (constant sheaf) and one obtains the formula $\chi(M)=e(T(M))[M]$. Next, if $\mathbf{D}=0$, i.e. $M$ is a complex manifold and $d_{\mathrm{F}}=\bar{\partial}$, then $T(M) / \mathrm{D}=T(M)$ is the complex tangent bundle and (24) reduces to the Hirzebruch-R-R formula $\chi(M, B)=$ $\operatorname{ch}(\mathrm{B}) \mathcal{T}(M)[M]$. 
In a large class of more special situations, the Riemann-Roch formula may be re-written in "simpler" terms. This is true, as an example, in the following case:

The compact, even-dimensional manifold $M$ is a fibre bundle over $L$ with fibre $B$ and projection $\pi: M \rightarrow L ; B$ is of dimension $2 d$ and $L$ of dimension $2 l$. In addition, $L$ is a complex manifold (of complex dimension $l$ ) and the bundle $\pi$ is oriented, so that the natural orientation of $L$ together with the one of the bundle yields an orientation of $M$. The preceding techniques thus apply to $M$ (and, of course, $L$ ). We also assume $B$ to be connected.

One then defines $F \subset T(M)_{C}$ by

$$
\mathbf{F}=(T \pi)^{-1}\left(T^{0,1}(L)\right)
$$

where $T \pi$ now denotes the extension of $T \pi: T(M) \rightarrow T(L)$ to $T(M)_{C}$. Clearly, F will satisfy the earlier conditions ([14, p. 249], [6, II, p. 233]) and, moreover, if we again write $\mathbf{F} \cap \overline{\mathbf{F}}=\mathbf{D}_{\mathbf{C}}$, then $\mathbf{D}$ is the vertical bundle of $\pi$ : $\mathbf{D}=\operatorname{ker}(T \pi) \subset T(M)$. For $y \in L$, we therefore have $\mathbf{D} \mid \pi^{-1}(y)=$ $T\left(\pi^{-1}(y)\right)$.

As before, we choose a summand $\mathbf{G}$ of $\mathbf{D}_{\mathbf{c}}$ in $\mathbf{F}$ e.g. by means of a hermitian structure on $T(M)_{\mathbf{C}}$, and we have $\overline{\mathbf{F}}=\mathbf{D}_{\mathbf{C}} \oplus \overline{\mathbf{G}}$. In this case, the natural map $T \pi_{*}: T(M)_{\mathbf{C}} \rightarrow \pi^{*} T(L)_{\mathbf{C}}$ induces an isomorphism of $\mathbf{G}$ onto $\pi^{*} T^{0,1}(L)$ and also of $\overline{\mathbf{G}}$ onto $\pi^{*} T^{1,0}(L)$. Since the latter is isomorphic to $\pi^{*} T(L), T(L)$ denoting the complex tangent bundle of $L$, we see that $\overline{\mathbf{G}} \approx \pi^{*} T(L)$.

Let now $\mathbf{E}$ be a holomorphic vector bundle over $L$. Then its pull-back $\pi^{*} \mathbf{E}$ is F-holomorphic: indeed, if $\left(V_{\alpha}\right)$ is an open covering of $L$ which trivializes $\mathbf{E}$ and if $\left(g_{\alpha \beta}\right)$ is the corresponding cocycle of holomorphic maps $g_{\alpha \beta}: V_{\alpha} \cap V_{\beta}$ $\rightarrow G L(E)$, then the covering given by the sets $U_{\alpha}=\pi^{-1}\left(V_{\alpha}\right)$ trivializes $\pi^{*} \mathbf{E}$ and the cocycle $\left(\pi^{*} g_{\alpha \beta}\right)$ gives the transition maps for $\pi^{*} \mathbf{E}$. Since $\pi^{*} g_{\alpha \beta}$ is constant on each $\left(U_{\alpha} \cap U_{\beta}\right) \cap \pi^{-1}(y)$, it is clear that $X \pi^{*} g_{\alpha \beta}=0$ for any local section $X$ of $\mathbf{D}$ (or $\mathbf{D}_{\mathbf{C}}$ ). If $X$ is a local section of $\mathbf{G}$, then $T \pi_{*} X$ is a section of $\pi^{*} T^{0,1}(L)$, thus is represented on the domain of $X$ by a map $Y$ into $T^{0,1}(L)$ which "lifts" $\pi$, i.e. one has $Y(x) \in T_{\pi(x)}^{0,1}(L)$. Therefore, $Y \pi^{*} g_{\alpha b}=0$ since the $g_{\alpha \beta}$ are holomorphic. This establishes the claim.

Note that we made use of the following fact: if $C(\mathbf{E}), \ldots$ denotes the sheaf of germs of smooth sections of $\mathbf{E}, \ldots$, then $C\left(\pi^{*} \mathbf{E}\right)=\pi^{*} \mathcal{C}(\mathbf{E})$, an identity which results immediately from the classical definition of the pull-back of a sheaf: if $\mathscr{F}$ is a sheaf over $L$, identified with the espace étalé it defines and if $p: \mathscr{F} \rightarrow L$ is the projection, then $\Gamma\left(U, \pi^{*} \mathscr{F}\right)$ for $U \subset M$ open is the set of all continuous maps $s: U \rightarrow \mathscr{F}$ which satisfy $p \circ s=\pi$ (the continuous lifts of $\pi$ into $\mathscr{F})$. Since in our case $\pi$ is continuous and open, we can define a second "pull-back" $\pi$ \# $\mathscr{F}$ by means of the presheaf $U \rightarrow \Gamma(\pi(U), \mathscr{F})$ on $M$. There is 
an obvious injection $\pi^{\#} \mathscr{F} \rightarrow \pi^{*} \mathscr{F}$, obtained by assigning to $t \in \Gamma(\pi(U), \mathscr{F})$ the section $t \cdot \pi$ of $\pi^{*} \mathscr{F}$ over $U$. Under this injection, $\pi^{\#} \mathscr{F}$ maps to the germs of fibre-constant sections of $\pi^{*} \mathcal{F}$.

REMARK. If $M$ is a principal $G$-bundle over $L$, then $\pi^{\#}$ can be obtained in a slightly different manner following [7, Chapter $\mathrm{V}]$ : in the notations of this reference $\pi^{\#}$ will be $\Gamma^{G} \circ \pi^{-1}$.

If again $\mathbf{E}$ is a holomorphic vector bundle over $L, \delta(\mathbf{E})$ denotes its sheaf of germs of holomorphic sections. Similarly, for an F-holomorphic vector bundle over $M, \delta_{\mathbf{F}}(\mathbf{B})$ is the sheaf of germs of $\mathbf{F}$-holomorphic sections of $\mathbf{B}$. With these notations, we obtain the following isomorphism:

$$
\pi^{\#} \mathcal{S}(\mathbf{E}) \cong \mathcal{S}_{\mathbf{F}}\left(\pi^{*} \mathbf{E}\right) \text {. }
$$

In other words: the canonical image of $\pi^{\#} \delta(\mathbf{E})$ in the smooth sections of $\pi^{*} \mathbf{E}$ is $\delta_{\mathbf{F}}\left(\pi^{*} \mathbf{E}\right)$ :

If $s \in \Gamma(\pi(U), \delta(\mathbf{E}))$ and if $X \in \Gamma(U, \mathbf{F})$, then $X(s \circ \pi)=0$ by the argument used above for $\pi^{*} g_{\alpha \beta}$. Conversely, let $s \in \Gamma\left(U, \pi^{*} E\right)$ satisfy $d_{F} s=$ 0 . Assuming that we are in local charts $U, \pi(U)$ over which the bundles $\pi^{*} \mathbf{E}$, E are trivial, this amounts to: $D s \cdot X=0$ for any smooth section $X$ of $F$ over $U$. In particular, this holds for $X \in \Gamma(U, D)$ and shows that $s$ is constant along the fibres of $\pi ; s$ is interpreted as a map $U \rightarrow \mathbf{E}$ over $\pi$, of course. Thus, $s=t \cdot \pi$ for some smooth $t: \pi(U) \rightarrow \mathrm{E}$ and we have to show that $t$ is holomorphic: Let $H$ be a section of $T^{0,1}(L)$ over $\pi(U)$. Then there is a unique $X \in \Gamma(U, C)$ with $T \pi_{*} X=\pi^{*} H$, i.e. with $D \pi \cdot X=H$, and now $0=D s \cdot X$ $=D t(D \pi \cdot X)=D t \cdot H$-and we are done.

As a consequence, the characteristic $\chi_{F}\left(M, \pi^{*} E\right)$ can also be written as $\chi\left(M, \pi^{\#} \delta(\mathbf{E})\right)$. At any rate it equals $\operatorname{ch}\left(\pi^{*} \mathbf{E}\right) e(\mathbf{D}) \mathcal{T}(T(M) / \mathbf{D})[M]$ by the Riemann-Roch formula. Since $\overline{\mathbf{G}} \cong T(M) / D$, an earlier remark shows that $\mathscr{T}(T(M) / \mathrm{D})=\pi^{*} \mathscr{T}(L), \mathcal{T}(L)$ the Todd class of the complex manifold $L$. Next, all cohomology classes in the formula consist of homogeneous components of even degree and therefore commute with each other; thus, their product may be written as $e(\mathbf{D}) \pi^{*}(\operatorname{ch}(\mathbf{E}) \mathcal{T}(L))$.

In order to evaluate this cohomology class, we use integration along the fibre, cf. [1, Chapter III] and [6, I, p. 307]. Denoting by $q$ the homomorphism of cohomology groups induced by integration along the fibre, the result is $e(D)^{\natural} \operatorname{ch}(\mathbf{E}) \mathcal{T}(L)[L]$ by [6], loc. cit. Observing that $e(D)$ induces the Euler class of the tangent bundle on each fibre $\pi^{-1}(y)$, one obtains $e(D)^{\natural}=\chi(B)$, the (ordinary) Euler-Poincare characteristic of the fibre $B$ of $\pi$. Next, by the Riemann-Roch theorem for compact complex manifolds, $\operatorname{ch}(E) \mathcal{T}(L)[L]=$ $\chi(L, \delta(\mathbf{E}))$.

Summarizing, we obtain 
Proposition 6. $M \rightarrow^{\pi} L$ is an oriented fibre bundle over the compact complex manifold $L$, with fibre $B$ of even dimension, $M$ compact, then for any holomorphic vector bundle $\mathbf{E}$ over $L$, we have

$$
\chi_{\mathbf{F}}\left(M, \pi^{*} \mathbf{E}\right)=\chi(B) \chi(L, \mathbf{E}) .
$$

Let, in particular, $\mathbf{E}=L \times \mathbf{C}$ be the trivial line bundle. Then $\delta(\mathbf{E})=\theta_{L}$, the sheaf of germs of holomorphic functions on $L$, and $\pi^{\#} \theta_{L}=\theta_{F}$. The corresponding characteristics therefore are the respective arithmetic genera and we have the

COROLLARY. The arithmetic genera of $M$ and $L$ are related by

$$
\chi_{a}(M)=\chi(B) \chi_{a}(L)
$$

or, in terms of the structural sheaves:

$$
\chi\left(M, \theta_{F}\right)=\chi(B) \chi\left(L, \theta_{L}\right) .
$$

In special cases, the right-hand side of (27) is "computable".

REMARK. One observes that the corollary is the analogue, in our context, of the multiplicativity of the arithmetic genus on holomorphic fibre bundles which is obtained by means of the Borel spectral sequence for the $\bar{\partial}$ cohomology, cf. [8, Appendix II]. The reason $\chi(B)$ replaces $\chi_{a}(B)$ in our formula is this: Due to the particular choice of $F$, the structure induced on each fibre is the entire (complexified) tangent bundle. Accordingly, the functions "holomorphic" with respect to this structure are the locally constant ones and these define the constant sheaf $\mathbf{C}$ on each fibre.

In a sense, Proposition 6 is "best possible" in the current situation. Indeed, note that we have shown that the sheaf of germs of F-holomorphic maps from $M$ into a complex vector space is of the form $\pi^{\#} \mathcal{F}, \mathcal{F}$ the sheaf of germs of holomorphic maps from $L$ into the given space. In particular, let $\mathcal{G}$ be the sheaf of germs of holomorphic maps $L \rightarrow G L(H), H$ a fixed complex vector space; then the corresponding sheaf $\mathcal{G}_{F}$ on $M$ simply is $\pi^{\#} \mathcal{G}$. This shows that the F-holomorphic vector bundles over $M$ with fibre $H$ are classified by $H^{1}\left(M, \pi^{\#} \mathcal{G}\right)$. Moreover, the map $\pi$ induces a bijection of $H^{1}\left(M, \pi^{\#} \mathcal{G}\right)$ onto $H^{1}(L, \mathcal{G})$ :

Since locally $M$ is a product over $L$, we see that every open covering of $M$ admits a refinement $\left(U_{\alpha}\right)$ with the property that $\pi\left(U_{\alpha} \cap U_{\beta}\right)=\pi\left(U_{\alpha}\right) \cap$ $\pi\left(U_{\beta}\right)$. Thus, let $\mathbf{H}$ be an $\mathbf{F}$-holomorphic vector bundle with fibre $E$, trivial over the open sets $U_{\alpha}$ of such a covering. Set $V_{\alpha}=\pi\left(U_{\alpha}\right)$. H is represented by a 1-cocycle $U_{\alpha} \cap U_{\beta} \rightarrow G L(E)$ consisting of F-holomorphic maps. Therefore, it is of the form $\left(\pi^{*} g_{\alpha \beta}\right)$ where the $g_{\alpha \beta}$ now are holomorphic maps $V_{\alpha} \cap V_{\beta} \rightarrow$ $G L(E)$; the cocycle $\left(g_{\alpha \beta}\right)$ represents a holomorphic bundle $\mathbf{E}$ over $L$ with fibre $E$ and it is clear that $\mathbf{H} \cong \pi^{*} \mathbf{E}$. This construction yields the desired bijection. 
Accordingly, up to isomorphisms, the F-holomorphic vector bundles over $M$ are precisely the pull-backs of holomorphic vector bundles over $L$ : The choice of F by (25) leads to the selection of these "natural" bundles. Moreover, known properties of holomorphic vector bundles (in particular: of line bundles) for suitably restricted complex manifolds $L$ will "lift" to the corresponding F-holomorphic bundles on $\boldsymbol{M}$.

One observes that the above construction $\mathbf{H} \rightarrow \mathbf{E}$ is compatible with the algebraic operations on vector bundles. Thus, denoting by $K_{\mathrm{F}}(M)$ the Grothendieck ring defined by the isomorphism classes of F-holomorphic vector bundles (i.e. locally free $\theta_{F}$-modules), $K_{\omega}(L)$ the one over the holomorphic vector bundles on $L$ (i.e. the locally free $\theta_{L}$-modules), the construction yields a ring homomorphism $\pi_{!}: K_{\mathrm{F}}(M) \rightarrow K_{\omega}(L)$. If $y \in K_{\mathrm{F}}(M)$ denotes the class of $\mathbf{H}$, then the right-hand side of (27) also may be written in the form $e(D))^{\natural} \operatorname{ch}\left(\pi_{!} y\right) \mathcal{T}(L)$ (evaluated on $L$ ), ch being extended to a homomorphism $K_{\omega}(L) \rightarrow H^{\bullet}(L, Q)$ as usual. In this sense, (27) vaguely resembles the Grothendieck form of R-R; in terms of cohomology classes, it reads as follows: $(e(\mathbf{D}) \operatorname{ch}(y) \mathscr{T}(\overline{\mathbf{G}}))^{\natural}=e(\mathbf{D})^{\natural} \operatorname{ch}\left(\pi_{!} y\right) \mathscr{T}(L)$ for $y \in K_{\mathrm{F}}(M)$. This is, of course, a trivial restatement of Proposition 5 and is an immediate consequence of the "proper" choice of F (i.e. according to formula (25)!). This choice also reduces the formula to a triviality in case $L$ is a point, as one readily sees. The question of a possible Riemann-Roch formula for "morphisms"-in the style of Grothendieck's theorem-will not be pursued further in this note. Analogously, formula (27) may be rewritten as

$$
\left(\operatorname{ch}(y) \mathscr{T}_{\mathbf{F}}(M)\right)^{\natural}=e(\mathbf{D})^{\natural} \operatorname{ch}\left(\pi_{!} y\right) \mathcal{T}(L)
$$

for $y \in K_{\mathbf{F}}(M)$.

5. The Frölicher spectral sequence. In addition, independently of any assumptions about ellipticity of $d_{\mathbf{F}}$, we are now going to establish the Frölicher spectral sequence relating the "Dolbeault" cohomologies $H^{\bullet}\left(M, \mathbb{Q}^{*}\right)$ and the (de Rham) cohomology $H^{\bullet}(M, \mathrm{C})$ with complex coefficients (cf. [5] for the complex-analytic case); it is interesting to observe that the first terms $E_{0}, E_{1}$ and $E_{2}$ of the spectral sequence have exactly the same interpretations as they do classically. We obtain all this by elementary computations, the key being Propositions 1,2 of $\$ 1$. In order to simplify the exposition, we introduce the following notations:

$C^{k}=\Gamma\left(\Omega^{k}(C)\right)$ is the vector space of smooth complex-valued $k$-forms on $M$ and $C=\bigoplus C^{k}=\Gamma(\Omega \cdot(C))$ is the space of all forms. The choice of $\mathbf{F}$ and a summand $\mathbf{K}$ defines the bigrading used earlier and we also set $C^{p, q}=$ $\Gamma\left(\Omega^{p, q}(\mathbf{C})\right)$; then $C^{k}=\bigoplus_{p+q=k} C^{p, q}$.

A decreasing filtration of $C^{k}$ is obtained as usual by setting 


$$
F^{p} C^{k}=\underset{\substack{r+s=k \\ r>p}}{\bigoplus} C^{r, s} \subset C^{k}
$$

For $p<0, F^{p} C^{k}=C^{k}$ and, moreover, $F^{p} C^{k}=0$ for large $p$, in fact: for $p>\operatorname{rank}(\mathbf{K})$. The decomposition $d=\partial+\tilde{\partial}+d_{\mathbf{F}}$ of the exterior derivative given in $\$ 1$ shows that

$$
d\left(F^{p} C^{k}\right) \subset F^{p} C^{k+1} .
$$

Setting $C_{p}=\bigoplus_{k>0} F^{p} C^{k}$, we obtain a decreasing filtration of $C$ with the following properties:

$$
\begin{gathered}
d\left(C_{p}\right) \subset C_{p} ; \quad C_{p} \cap C^{q}=F^{p} C^{q}=0 \text { for } p>q \\
C_{p}=0 \quad \text { for } p>\operatorname{rank}(\mathbf{K}) .
\end{gathered}
$$

Lastly, the single grading (by total degree) of $C$ and the filtration are compatible: if $x=\sum x_{k} \in C_{p}$ for some $p$, then $x_{k} \in C_{p}$ for every $k$.

Thus, the usual conditions are satisfied and the bigrading of $C$ defines a spectral sequence $\left(E_{r}^{p, r}\right)$ which will be regular. Since some complications in the computations arise from the fact that only $d_{\mathbf{F}}^{2}=0$, in general, while $\partial+\tilde{\partial}$ does not have the same property, we are going to give some details of the arguments used:

Standard arguments show that $Z_{r}^{p, q}=\left\{x \in F^{p} C^{p+q} \mid d x \in F^{p+r} C^{p+q+1}\right\}$ and that $B_{r}^{p, q}=d\left(F^{p-r} C^{p+q-1}\right) \cap F^{p} C^{p+q}$; moreover

$$
F^{p} C^{p+q}=C^{p, q} \oplus F^{p+1} C^{p+q} \text {. }
$$

Proposition 7. $E_{0}^{p, q}=C^{p, q}$.

Indeed, $E f^{p, q}=\left(C_{p} \cap C^{p+q}\right) /\left(C_{p+1} \cap C^{p+q}\right)=F^{p} C^{p+q} / F^{p+1} C^{p+q} \cong$ $C^{p, q}$ by (28).

PROPOSITION 8. $Z_{1}^{p, q}=\operatorname{ker}\left(C^{p, q} \stackrel{d_{\mathrm{F}}}{\rightarrow} C^{p, q+1}\right) \oplus F^{p+1} C^{p+q}$.

To simplify the notations, let us write $\operatorname{ker}\left(d_{\mathrm{F}} \mid C^{p, q}\right)$ for $\operatorname{ker}\left(C^{p, q} \stackrel{d_{\mathrm{F}}}{\rightarrow} C^{p, q+1}\right)$. Now, by definition, $Z_{1}^{p, q}=\left\{x \in F^{p} C^{p+q} \mid d x \in F^{p+1} C^{p+q+1}\right\}$. Write $F^{p} C^{p+q}$ $=C^{p, q} \oplus F^{p+1} C^{p+q}$. We know that $d\left(F^{p+1} C^{p+q}\right) \subset F^{p+1} C^{p+q+1}$; the condition $x \in Z_{1}^{p, q}$ for $x=a+b$ with $a \in C^{p, q}, b \in F^{p+1} C^{p+q}$, therefore reduces to $d a \in F^{p+1} C^{p+q+1}$. Now $d a=d_{\mathrm{F}} a+\partial a+\tilde{\partial} a$ and, since $\partial$ is of type $(1,0)$, $\tilde{\partial}$ of type $(2,-1), \partial a+\tilde{\partial} a \in F^{p+1} C^{p+q+1}$. Thus, $a+b$ is in $Z_{1}^{p, q}$ if and only if $d_{\mathrm{F}} a=0$. This immediately implies the result.

PROPOSITION 9. $B_{b}^{p, q}+Z_{\delta}^{p+1, q-1}=d_{\mathrm{F}}\left(C^{p, q-1}\right) \oplus F^{p+1} C^{p+q}$.

By earlier remarks, $Z_{0}^{p+1, q-1}=\left\{x \in F^{p+1} C^{p+q} \mid d x \in F^{p+1} C^{p+q+1}\right\}=$ $F^{p+1} C^{p+q}$. On the other hand, $B_{0}^{p, q}=d\left(F^{p} C^{p+q-1}\right) \cap F^{p} C^{p+q}=$ $d\left(F^{p} C^{p+q-1}\right)=d\left(C^{p, q-1} \oplus F^{p+1} C^{p+q-1}\right)$ and $d\left(F^{p+1} C^{p+q-1}\right) \subset F^{p+1} C^{p+q}$. Next, if $x \in C^{p, q-1}$, then $d x=d_{\mathrm{F}} x+\partial x+\tilde{\partial x}$ shows that $\partial x+\tilde{\partial x}$ already 
lies in $F^{p+1} C^{p+q}$, so that $B_{0}^{p, q}=d_{\mathrm{F}}\left(C^{p, q-1}\right) \oplus H$ with $H \subset F^{p+1} C^{p+q}$. With this, the assertion is immediate.

Combining the last two propositions, we conclude:

Proposition 10. $\operatorname{ker}\left(d_{\mathrm{F}} \mid C^{p, q}\right) / d_{\mathrm{F}}\left(C^{p, q-1}\right) \cong E_{1}^{p, q}$.

Together with the earlier results, this shows, in particular, that

$$
E_{1}^{p, q} \cong H^{q}\left(M, \mathbb{Q}^{p}\right),
$$

the "Dolbeault cohomology" of $(M, \mathbf{F}, \mathbf{K})$. Thus, the $E_{0}$ and $E_{1}$-terms of the spectral sequence obtained here coincide with the corresponding terms of Frölicher's spectral sequence in the complex-analytic case.

In order to determine $E_{2}^{p, q}$, recall from Proposition 2 that $d_{\mathrm{F}} \partial+\partial d_{\mathrm{F}}=0$, so that $\partial$ induces a map

$$
\partial_{1}: \operatorname{ker}\left(d_{\mathrm{F}} \mid C^{p, q}\right) / d_{\mathrm{F}}\left(C^{p, q-1}\right) \rightarrow \operatorname{ker}\left(d_{\mathrm{F}} \mid C^{p+1, q}\right) / d_{\mathrm{F}}\left(C^{p+1, q-1}\right) ;
$$

this map now satisfies $\partial_{1}^{2}=0$ :

We know that $\partial^{2}+d_{\mathrm{F}} \tilde{\partial}+\tilde{\partial} d_{\mathrm{F}}=0$. Thus, if $x \in C^{p, q}$ satisfies $d_{\mathrm{F}} x=0$, then $\partial^{2} x=-d_{F} \tilde{\partial} x \in d_{F}\left(C^{p+2, q-1}\right)$, whence $\partial_{1}^{2}=0$.

On the other hand, $d$ induces a map $d_{1}: E_{1}^{p, q} \rightarrow E_{1}^{p+1, q}, d_{1}^{2}=0$, and the $E_{2}$-term is the cohomology of the complex obtained in this manner. We include here the explicit verifications since this will also yield the desired interpretation of $E_{2}$ :

Let us show that $d\left(Z_{1}^{p, q}\right) \subset Z_{1}^{p+1, q}$ : Using Proposition $8, z \in Z_{1}^{p, q}$ may be written in the form $z=z_{p, q}+w$ with $d_{\mathrm{F}} z_{p, q}=0$ and $w \in F^{p+1} C^{p+q}$. Set $w=w_{p+1}+h, w_{p+1} \in C^{p+1, q-1}$ and $h \in F^{p+2} C^{p+q}$. In this case, $d\left(z_{p, q}+\right.$ $w)=\partial z_{p, q}+\tilde{\partial} z_{p, q}+d_{F} w_{p+1}+\partial w_{p+1}+\tilde{\partial} w_{p+1}+d h$ where $d h \in F^{p+2} C^{p+q+1}$ as well as $\partial w_{p+1}, \partial w_{p+1} \in F^{p+2} C^{p+q+1}$; similarly, $\tilde{z}_{p, q} \in F^{p+2} C^{p+q+1}$. Also, $d_{F} w_{p+1} \in C^{p+1, q}$ with $d_{F}\left(d_{F} w_{p+1}\right)=0$ and, lastly, $\partial z_{p, q} \in C^{p+1, q}$ and $d_{F} \partial z_{p, q}$ $=-\partial d_{\mathrm{F}} z_{p, q}=0$. Thus, $\partial z_{p, q}+d_{\mathrm{F}} w_{p+1} \in \operatorname{ker}\left(d_{\mathrm{F}} \mid C^{p+1, q}\right)$ and the remaining terms lie in $F^{p+2} C^{p+q+1}$.

The calculation actually shows more:

$$
d\left(z_{p, q}+w\right) \equiv \partial z_{p, q} \bmod d_{\mathbf{F}}\left(C^{p+1, q-1}\right)
$$

and this will enable us below to give a more concrete interpretation of $E_{2}$.

Next, let $d_{\mathrm{F}} c_{p, q-1}+u \in d_{\mathrm{F}}\left(C^{p, q-1}\right) \oplus F^{p+1} C^{p+q}$ and again write $u=u_{p+1}$ $+k$ with $u_{p+1} \in C^{p+1, q-1}$ and $k \in F^{p+2} C^{p+q}$. Then $d k \in F^{p+2} C^{p+q+1}$ and the remaining part of $d\left(d_{\mathrm{F}} c_{p, q-1}+u\right)$ is: $\partial d_{\mathrm{F}} c_{p, q-1}+\tilde{\partial} d_{\mathrm{F}} c_{p, q-1}+d_{\mathrm{F}} u_{p+1}+$ $\partial u_{p+1}+\tilde{\partial} u_{p+1}$; evidently, the last two terms are in $F^{p+2} C^{p+q+1}$ and $d_{F} u_{p+1} \in$ $d_{\mathbf{F}}\left(C^{p+1, q-1}\right)$. The first term is $-d_{\mathbf{F}} \partial c_{p, q-1} \in d_{\mathbf{F}}\left(C^{p+1, q-1}\right)$ and the second one may be rewritten as $-d_{F} \tilde{\partial} c_{p, q-1}-\partial^{2} c_{p, q-1} \in C^{p+2, q-1}$; all this now shows $d\left(B_{0}^{p, q}+Z_{0}^{p+1, q-1}\right) \subset B_{0}^{p+1, q}+Z_{0}^{p+2, q-1}$.

With this, $d_{1}$ is determined. Moreover, the first step shows that the following diagram commutes: 


$$
\begin{array}{ccc}
\operatorname{ker}\left(d_{\mathrm{F}} \mid C^{p, q}\right) / d_{\mathrm{F}}\left(C^{p, q-1}\right) & \rightarrow & E_{1}^{p, q} \\
\partial_{1} \downarrow & & \downarrow d_{1} \\
\operatorname{ker}\left(d_{\mathrm{F}} \mid C^{p+1, q}\right) / d_{\mathrm{F}}\left(C^{p+1, q-1}\right) & \rightarrow & E_{1}^{p+1, q}
\end{array}
$$

where the horizontal arrows denote the isomorphisms of Proposition 10.

Combining this with (29), we see that once again we obtain the complex

$$
\cdots \stackrel{\partial_{1}}{\rightarrow} H^{q}\left(M, \mathbb{Q}^{p}\right) \stackrel{\partial_{1}}{\rightarrow} H^{q}\left(M, \mathbb{Q}^{p+1}\right) \stackrel{\partial_{1}}{\rightarrow} \ldots
$$

whose cohomology groups are precisely the terms $E_{2}^{p, q}$ of the spectral sequence, cf. [5].

Since the spectral sequence is regular, we have $E_{r}^{p, q}=E_{\infty}^{p, q}$ for large $r$ (in fact: for $r>\max (p, q+1))$. Moreover, the "total cohomology" $\mathbf{H}(C)$ is given by

$$
H^{p}(C)=\left(\operatorname{ker}(d) \cap C^{p}\right) / d\left(C^{p-1}\right)
$$

and is the de Rham cohomology of $M$ with complex coefficients. We obtain a decreasing filtration of $H^{p}(C)$ in the usual way by defining $H^{p}(C)_{n}=$ $\pi\left(\operatorname{ker}(d) \cap C^{p} \cap C_{n}\right), \pi$ the canonical map $\operatorname{ker}(d) \cap C^{p} \rightarrow H^{p}(C)$. As usual,

$$
E_{\infty}^{p, q}=H^{p+q}(C)_{p} / H^{p+q}(C)_{p+1} \text {. }
$$

Letting $b_{p}=\operatorname{dim}\left(H^{p}(C)\right)$, this yields as a corollary the formula

$$
b_{p}=\sum_{r+s=p} \operatorname{dim}_{\mathbf{C}}\left(E_{\infty}^{r, s}\right) \quad(r, s \geqslant 0)
$$

Choosing $N>p+1$, we have $E_{N}^{r, s}=E_{\infty}^{r, s}$ for $r+s=p$, so that we may use the groups $E_{N}^{r, s}$ in this last formula. Since evidently $\operatorname{dim}_{\mathbf{C}}\left(E_{a}^{r, s}\right) \leqslant \operatorname{dim}_{C}\left(E_{a-1}^{r, s}\right)$, we conclude the

PROPOSITION 11. $b_{p} \leqslant \sum_{r+s=p} \operatorname{dim}_{\mathbf{C}}\left(H^{s}\left(M, \mathbb{Q}^{r}\right)\right)$.

Assuming the Betti numbers $b_{p}$ and $b_{p, q}=\operatorname{dim}_{C}\left(E_{\infty}^{p, q}\right)$ finite, the EulerPoincaré characteristic of $M$ is $\chi(M)=\Sigma(-1)^{p} b_{p}=\Sigma(-1)^{p+q} b_{p, q}$ by the formula following (32). On the other hand, $E_{r+1}$ is the cohomology of $\left(E_{r}, d_{r}\right)$ and $d_{r}$ is of total degree 1 , so that elementary linear algebra shows that

$$
\sum(-1)^{p+q} \operatorname{dim}_{\mathbf{C}}\left(E_{r}^{p, q}\right)=\sum(-1)^{p+q} \operatorname{dim}_{\mathbf{C}}\left(E_{r+1}^{p, q}\right) .
$$

Thus, (31) implies

Proposition 12. $\chi(M)=\Sigma(-1)^{p+q} \operatorname{dim}_{\mathrm{C}}\left(H^{q}\left(M, \mathbb{Q}^{p}\right)\right)$.

The last two propositions are the analogues of Frölicher's two theorems in [5]. Recall that if $M$ is compact, then the $H^{q}\left(M, Q^{p}\right)$ are finite-dimensional; thus, in this case Proposition 12 will hold. 
6. Serre duality. In this section, the manifold $M$ is assumed to be oriented and countable at infinity (e.g.: connected). We wish to investigate possible analogues of the Serre duality theorem, established for complex manifolds in [18], cf. also [22, p. 179]. In order to obtain such a theorem without any compactness assumption on $M$, we attempt to follow Serre's original approach which, in addition to differential forms, uses currents:

First of all, if $\mathbf{B}$ is an F-holomorphic vector bundle, $\delta_{\mathbf{F}}(\mathbf{B})$ the sheaf of germs of F-holomorphic sections, $\theta_{\mathbf{F}}=\delta_{\mathbf{F}}$ the sheaf of germs of Fholomorphic functions, then

$$
\Omega^{p, q}(\mathbf{B})=\mathcal{\delta}_{\mathbf{F}}(\mathbf{B}) \otimes_{\Theta_{\mathbf{F}}} \Omega^{p, q}, \quad \Omega^{p, q}=\Omega^{p, q}(\mathbf{C}) .
$$

This is proved in the usual manner, using the existence of local Fholomorphic frames of $\mathbf{B}$. The same argument also shows that $\delta_{\mathbf{F}}(\mathbf{B})$ is locally free of rank equal to $\operatorname{rank}(\mathbf{B})$ over $\Theta_{\mathbf{F}}$. Moreover, under the identification (33), $d_{\mathrm{F}}: \Omega^{p, q}(\mathbf{B}) \rightarrow \Omega^{p, q+1}(\mathbf{B})$ is given by $1 \otimes d_{\mathrm{F}}$, from which one concludes again that the complex $\left(\Omega^{p, q}(\mathbf{B}), d_{\mathbf{F}}\right)_{q}$ is acyclic (since $\mathcal{S}_{\mathbf{F}}(\mathbf{B})$ is locally free!).

In the sequel, we shall also use the following notations: $n=\operatorname{rank}(\mathbf{F})$, $N=\operatorname{dim}(M), n^{*}=N-n=\operatorname{rank}(\mathbf{K})$. In this case, $\Omega^{n^{*}, n}=\Omega^{N}$ is the sheaf of smooth $\mathbf{C}$-valued forms of maximal degree on $M$, an invertible $\mathcal{C}$-module. If $U \subset M$ is open, $\Gamma_{c}(U, \ldots)$ denotes the module of sections with compact support contained in $U$ (of a sheaf or a bundle).

Let $\mathbf{V}$ be a complex vector bundle, $U \subset M$ open. The vector space $\Gamma(U, \mathbf{V})=\Gamma(U, \delta(\mathbf{V}))$ will be given the topology of local uniform convergence of the sections and their derivatives ( $C^{\infty}$-topology): with this, it will be a Fréchet space even when $U=M, M$ being countable at infinity. $\Gamma_{c}(U, \delta(V))$ will be considered as the inductive limit of the Fréchet spaces $\Gamma(U, \delta(\mathbf{V}))_{C}$ of sections of $\mathbf{V}$ with support in the compact $C \subset U$; with this topology, $\Gamma_{c}(U, \delta(V))$ is an $L F$-space. The topological duals will be denoted by $\Gamma(U, \delta(\mathbf{V}))^{\prime}$ and $\Gamma_{c}(U, \delta(\mathbf{V}))^{\prime}$, resp., and no topologies will be specified on these spaces.

The sheaf $\mathcal{K}^{p, q}$ of germs of currents of type $(p, q)$ now is defined by the presheaf $U \rightarrow \Gamma_{c}\left(U, \Omega^{n^{*}-p, n-q}\right)^{\prime}$ on $M:$ for $V \subset U$, we have $\Gamma_{c}(V, \delta(V)) \rightarrow$ $\Gamma_{c}(U, \delta(V))$ by means of the "extension by zero" (trivial extension) of the sections and this yields a continuous injection whose transpose is the required "restriction map". One can show that the presheaf obtained in this manner is (canonically isomorphic to) $U \rightarrow \Gamma\left(U, \mathscr{K}^{p, q}\right)$ with which it will be identified. Given a current $T$ and a form $\omega$, we write $\langle T, \omega\rangle$ for the value of $T$ on $\omega$, sometimes denoted by $T[\omega]$ in the literature, cf. e.g. [16], [3]; our notations agree with those of [19].

The sheaf $\mathscr{K}^{0,0}$ plays a special rôle: it is the sheaf of germs of distributions on $M$, defined by the presheaf $U \rightarrow \Gamma_{c}\left(U, \Omega^{N}\right)^{\prime}$; this definition allows for the 
standard interpretation of a function as a distribution: for a function $f$ and an $N$-form $\omega$ with compact support, $\langle f, \omega\rangle$ is defined to be $\int f \omega$. Since $M$ is oriented, integration of forms is meaningful at least if the support of the form is compact. More generally, if $\alpha$ is any smooth form of type $(p, q)$ on $U$, then it defines a current $T_{\alpha}$ by $\left\langle T_{\alpha}, \omega\right\rangle=\int \alpha \wedge \omega$ and this construction yields an injective $\mathcal{C}$-homomorphism

$$
\Omega^{p, q} \rightarrow \mathcal{K}^{p, q} .
$$

We define the operator $\tilde{d}_{\mathbf{F}}$ on $\mathcal{K}^{p, q}$ in such a way that it extends $d_{\mathbf{F}}$ as defined on $\Omega^{p, q}$ :

If $T$ is a current of type $(p, q)$ on $U$, we set

$$
\left\langle\tilde{d}_{\mathbf{F}} T, \omega\right\rangle=(-1)^{p+q+1}\left\langle T, d_{\mathbf{F}} \omega\right\rangle
$$

for any form $\omega$ of type $\left(n^{*}-p, n-q\right)$ with compact support in $U$. One clearly has $\tilde{d}_{\mathrm{F}}^{2}=0$, so that for any fixed $p,\left(\mathcal{F}^{p, q}, \tilde{d}_{\mathrm{F}}\right)_{q}$ is a sheaf complex. In order to simplify the notations, we sometimes write $d_{F}$ in lieu of $\tilde{d}_{F}$. One verifies easily that if $\alpha$ is a form of type $(p, q)$ on $U$, then $\tilde{d}_{\mathrm{F}} T_{\alpha}=T_{d_{\mathrm{F}} \alpha}$, as claimed.

If $T$ is a current of type $(p, q)$ and $\alpha$ is a form of type $(r, s)$ on $U$, we define the current $T \wedge \alpha$ of type $(p+r, q+s)$ by $\langle T \wedge \alpha, \omega\rangle=\langle T, \alpha \wedge \omega\rangle$ and $\alpha \wedge T$ by $(-1)^{(p+q)(r+s)} T \wedge \alpha$, cf. [16]; this extension of exterior multiplication yields homomorphisms

$$
\mathcal{K}^{p, q} \otimes_{\mathcal{C}} \Omega^{r, s} \rightarrow \mathcal{K}^{p+r, q+s}
$$

and, in particular, there are natural homomorphisms

$$
\mathscr{K}^{0,0} \otimes_{e} \Omega^{p, q} \rightarrow \mathcal{K}^{p, q} .
$$

In these last two assertions, multiplication of a current $T$ by a function $f$ is defined, as a special case of exterior multiplication, by $\langle f T, \omega\rangle=\langle T, f \omega\rangle$.

The map of (37) is described explicitly as follows: if $D$ is a distribution on $U, \omega$ a form of type $(p, q)$, then $\langle D \wedge \omega, \beta\rangle=\langle D, \omega \wedge \beta\rangle$. In particular, for a function $h$ on $U$, one has $\langle D \wedge \omega, h \beta\rangle=\langle D, h \omega \wedge \beta\rangle$. Now, suppose that $U$ is small enough so that $\mathbf{K}$ and $\mathbf{F}$ are trivial over $U$ with fibres $K, F$, respectively. Then $\Gamma\left(U, \Omega^{p, q}\right)$ is identified with the space of smooth maps $U \rightarrow\left(\bigwedge^{p} K \otimes \bigwedge^{q} F\right)^{*}, \Gamma_{c}\left(U, \Omega^{n^{*}-p, n-q}\right)$ with the space of smooth maps $U \rightarrow$ $\left(\wedge^{n^{*}-p} K \otimes \bigwedge^{n-q} F\right)^{*}$ with compact support and, lastly, $\Gamma_{c}\left(U, \Omega^{N}\right)$ with the smooth maps $U \rightarrow\left(\bigwedge^{N} H\right)^{*}$ with compact support. The C-vector spaces $\left(\bigwedge^{p} K \otimes \bigwedge^{q} F\right)^{*}$ and $\left(\bigwedge^{n^{*}-p} K \otimes \bigwedge^{n-q} F\right)^{*}$ have the same dimension $\left(\begin{array}{l}n^{*} \\ p\end{array}\right)\left(\begin{array}{l}n \\ q\end{array}\right)$ and by standard linear algebra, there exist bases $\left(\omega_{i}\right),\left(\beta_{i}\right)$ of these spaces with

$$
\begin{aligned}
& \omega_{i} \wedge \beta_{i} \neq 0, \\
& \omega_{i} \wedge \beta_{k}=0 \text { if } i \neq k,
\end{aligned}
$$

where $\omega_{i} \wedge \beta_{j} \in\left(\wedge^{N} H\right)^{*}$ (under the usual identifications). Thus, given $i$, any 
element of $\Gamma_{c}\left(U, \Omega^{N}\right)$ is uniquely written in the form $h \omega_{i} \wedge \beta_{i}$ with $h \in$ $C_{c}(U)$. Moreover, $h \beta_{i}$ is a form of type $\left(n^{*}-p, n-q\right)$ with compact support and we now define a distribution $D_{i}$, given the current $T$ of type $(p, q)$, by

$$
\left\langle D_{i}, h \omega_{i} \wedge \beta_{i}\right\rangle=\left\langle T, h \beta_{i}\right\rangle \text {. }
$$

Since $\omega_{i} \wedge \beta_{i} \neq 0$ on $U$, every $N$-form with compact support is of the form $h \omega_{i} \wedge \beta_{i}$, so that the definition is meaningful. Moreover, one readily verifies that $T=\Sigma D_{i} \wedge \omega_{i}$. In other words: the natural map of (37) is an isomorphism (cf. [16, p. 42], for the classical case).

Next, we wish to determine the kernel of $\tilde{d}_{F}$ in $\mathcal{K}^{p, 0}$ : This is a local question and therefore, we may apply the Nirenberg theorem once again and reduce the situation to the following one: the parameter space $H$ of $M$ is written as $H=L \times\left(D \times E_{0}\right), D \times E_{0}=E$, and we are dealing with an open subset of the form $V_{1} \times(U \times V)$, where $V_{1}, U$ and $V$ are open convex neighborhoods of 0 in $L, D$ and $E_{0}$. In addition, $E_{0}$ is complex so that $U \times V$ has coordinates $\left(x_{1}, \ldots, x_{d} ; z_{1}, \ldots, z_{e}\right)$ with $d=\operatorname{dim}_{\mathrm{R}}(D), 2 e=\operatorname{dim}_{\mathrm{R}}\left(E_{0}\right)$. Setting $d z_{\alpha}=d z_{i_{1}} \wedge \cdots \wedge d z_{i_{p}}, i_{1}<\cdots<i_{p}$, a current of type $(p, 0)$ on $V_{1} \times(U \times V)$ then is written in the form

$$
T=\sum_{\alpha} D_{\alpha} \wedge d z_{\alpha}
$$

where each $D_{\alpha}$ is a distribution on $V_{1} \times(U \times V)$. Moreover, as earlier, $\tilde{d}_{\mathrm{F}}$ goes over to the operator $d_{D}+\bar{\partial}$ where $d_{D}$ is exterior differentiation on $U$. Accordingly, $\tilde{d}_{\mathbf{F}} T$ becomes

$$
\left(d_{D}+\bar{\partial}\right) T=-\sum_{\alpha} d_{D} D_{\alpha} \wedge d z_{\alpha}-\sum_{\alpha} \bar{\partial} D_{\alpha} \wedge d z_{\alpha}
$$

From this we conclude

$$
d_{D} D_{\alpha}=0, \quad \bar{\partial} D_{\alpha}=0,
$$

since $d_{D} D_{\alpha}, \bar{\partial} D_{\alpha}$ only involve the forms $d x_{i}, d \bar{z}_{j}$, respectively.

Assume now that $L=0$. This amounts to $E=H$, i.e. to the assumption that $F$ should be elliptic, as noted earlier. In this case, (39) implies that $D_{\alpha}$ is independent of $x \in U$, i.e. is a distribution on $V$ which is $\bar{\partial}$-closed and hence is a holomorphic function, cf. [16], [3]. As a consequence, $T$ is a differential form. Summarizing, we obtain the

THEOREM $1^{\prime}$. Assume that $\mathbf{F}$ is elliptic. Then $\operatorname{ker}\left(\tilde{d}_{\mathrm{F}} \mid \mathcal{K}^{p, 0}\right)=\mathbb{Q}^{p}$ and therefore, for each $p \geqslant 0,\left(\mathcal{K}^{p, q}, \tilde{d}_{\mathrm{F}}\right)$ is a fine resolution of $\mathbb{Q}^{p}$. Thus,

$$
H^{q}\left(M, \mathbb{Q}^{p}\right)=H^{q}\left(\left(\Gamma\left(\mathcal{K}^{p \cdot}\right), \tilde{d}_{\mathrm{F}}\right)\right) .
$$

Note that the ellipticity of $\mathbf{F}$ also is necessary for Theorem $1^{\prime}$ : indeed, if $L \neq 0$ in the above notations, then there are distributions $S$ on $V_{1} \times(U \times$ $V$ ) satisfying $\tilde{d}_{F} S=0$ without being functions: it suffices to take a decom- 
posable distribution $S=D_{1} \otimes D_{2}, D_{1}$ on $V_{1}$ and $D_{2}$ on $U \times V$. Then $\left(d_{D}+\right.$ $\bar{\partial})\left(D_{1} \otimes D_{2}\right)=D_{1} \otimes\left(d_{D}+\bar{\partial}\right) D_{2}$ (cf. [19, Chapter 40]) and this vanishes already if $\left(d_{D}+\bar{\partial}\right) D_{2}=0$ while $D_{1}$ is arbitrary. All this means that $F$ is elliptic if and only if $\tilde{d}_{F}$ is "hypoelliptic" in the sense that its kernel in the distributions consists of functions.

Let again $B$ be an F-holomorphic vector bundle over $M$. Following [18], we define the sheaves of germs of B-valued currents of type $(p, q)$ to be

$$
\mathcal{K}^{p, q}(\mathbf{B})=\mathcal{S}_{\mathbf{F}}(\mathbf{B}) \otimes_{\Theta_{\mathbf{F}}} \mathcal{K}^{p, q} \text {. }
$$

Under the embedding $\Omega^{p, q}(\mathbf{B}) \subset \mathcal{K}^{p, q}(\mathbf{B})$ induced by $\Omega^{p, q} \subset K^{p, q}$, the operator $\tilde{d}_{\mathbf{F}}$ defined as earlier again extends $d_{\mathbf{F}}$ to the currents and, moreover, now is identified with $1 \otimes \tilde{d}_{\mathbf{F}}$ under (40) since the tensor product is taken over $\theta_{\mathbf{F}}$ (as was the case in (33)). Theorem $1^{\prime}$ now immediately implies the

THEOREM $2^{\prime}$. If $\mathbf{F}$ is elliptic, then for each $p \geqslant 0,\left(\mathscr{K}^{p, q}(\mathbf{B}), \tilde{d}_{\mathbf{F}}\right)_{q>0}$ is a fine resolution of $\mathbb{Q}^{p}(\mathbf{B})$.

REMARK. Since so far we have been using fine resolutions to establish the cohomology theorems, the corresponding theorems will still hold for any "paracompactifying" support family $\Phi$, the cohomology $H_{\Phi}$ with supports in $\Phi$ and the section functor $\Gamma_{\Phi}$. In addition to the case already treated here, we shall need the cohomologies $H_{c}$ with compact supports; the preceding results thus still hold for $H_{c}$.

Given the F-holomorphic vector bundle B, let us introduce the following notations:

$$
\begin{aligned}
& A^{p, q}(\mathbf{B})=\Gamma\left(\Omega^{p, q}(\mathbf{B})\right) \\
& K^{p, q}(\mathbf{B})=\Gamma\left(\mathcal{K}^{p, q}(\mathbf{B})\right) \\
& K_{c}^{p, q}(\mathbf{B})=\Gamma_{c}\left(\mathcal{K}^{p, q}(\mathbf{B})\right) .
\end{aligned}
$$

The vector space $A^{p, q}(\mathbf{B})$ is given its $C^{\infty}$-topology which makes it into a Fréchet space. These notations essentially agree with those of [18] and we follow Serre's arguments for the remainder of this section:

The duality between $\mathbf{B}$ and $\mathbf{B}^{*}$ induces a pairing $\delta_{\mathbf{F}}(\mathbf{B}) \times \delta_{\mathbf{F}}\left(\mathbf{B}^{*}\right) \rightarrow \mathcal{\theta}_{\mathbf{F}}$, i.e. a homomorphism $\mathfrak{S}_{\mathbf{F}}(\mathbf{B}) \otimes \mathcal{S}_{\mathbf{F}}\left(\mathbf{B}^{*}\right) \rightarrow \mathcal{O}_{\mathbf{F}}$. On the other hand, there is the homomorphism of (36). Combining these, there results a homomorphism

$$
\varepsilon: \Omega^{p, q}(\mathbf{B}) \otimes_{\mathcal{O}_{\mathbf{F}}} \mathcal{K}^{r, s}\left(\mathbf{B}^{*}\right) \rightarrow \mathcal{K}^{p+r, q+s},
$$

cf. [18]. Locally, if $\omega=s \otimes \beta$ (cf. (33)!) and $S=t \otimes T$ (cf. (40)!), then $\varepsilon(\omega \otimes S)$ acts on $\alpha$ by

$$
\langle\varepsilon(\omega \otimes S), \alpha\rangle=\langle t, s\rangle\langle\beta \wedge T, \alpha\rangle,
$$

$\langle t, s\rangle$ being the function given by $t, s$ and the pairing of $\mathbf{B}^{*}$ and $\mathbf{B}$. Following [18], we denote $\varepsilon(\omega \otimes S)$ by $\omega \wedge S$. 
In particular, if $\omega$ is of type $(p, q)$ on $M$, i.e. in $A^{p, q}(\mathrm{~B})$, and if $T$ is in $K_{c}^{n^{*}-p, n-q}\left(B^{*}\right)$, then $\omega \wedge T$ is a current of maximal type $\left(n^{*}, n\right)$ with compact support and therefore can be integrated over $M$; we set

$$
\langle\langle\omega, T\rangle\rangle=\int \omega \wedge T \quad(=\langle\omega \wedge T, 1\rangle)
$$

1 being the constant function equal to 1 on $M$. Since the support of $\omega \wedge T$ is compact, this makes sense by, e.g. [17, p. 42]. Defining the linear form $L_{T}$ on $A^{p, q}(\mathrm{~B})$ by: $\left\langle L_{T}, \omega\right\rangle=\langle\langle\omega, T\rangle\rangle$, one obtains the

LEMMA. The map $T \rightarrow L_{T}$ is an isomorphism of $K_{c}^{n^{*}-p, n-q}\left(\mathrm{~B}^{*}\right)$ onto the topological dual of $A^{p, q}(\mathbf{B})$.

Proof. [18, Proposition 4 and footnote 5 on p. 18].

If one identifies the two vector spaces under this map, then the transpose of the (obviously continuous) linear map $d_{\mathrm{F}}: A^{p, q}(\mathrm{~B}) \rightarrow A^{p+q+1}(\mathrm{~B})$ is identified with $(-1)^{p+q+1} \tilde{d}_{\mathbf{F}}$.

With this, it becomes evident that the arguments of $[18, \S 10]$, carry over to the present case, establishing as a main result the

Theorem 6 (Serre Duality Theorem). Assume that $M$ is countable at infinity and that $\mathbf{F}$ is elliptic. Let $\mathbf{B}$ be an $\mathbf{F}$-holomorphic vector bundle over $M$.

If in the sequence $A^{p, q-1}(\mathrm{~B}) \rightarrow A^{p, q}(\mathrm{~B}) \rightarrow A^{p, q+1}(\mathrm{~B})$, the two maps $d_{\mathrm{F}}$ are homomorphisms of Fréchet spaces, then there is a canonical isomorphism

$$
H^{q}\left(M, \mathbb{Q}^{p}(\mathbf{B})\right)^{\prime} \cong H_{c}^{n-q}\left(M, \mathbb{Q}^{n^{*}-p}\left(\mathbf{B}^{*}\right)\right)
$$

By a homomorphism, we here mean a linear map which is continuous and open onto its image, i.e. which identifies the quotient mod its kernel both linearly and topologically with its image. In the category of Fréchet spaces, this amounts to saying that the map is continuous with a closed range.

We conclude, as usual:

COROllary. If, in addition to the assumptions of the theorem, $M$ is compact, then the vector spaces $H^{q}\left(M, \mathbb{Q}^{p}(\mathrm{~B})\right)$ and $H^{n-q}\left(M, \mathbb{Q}^{n^{*}-p}\left(\mathrm{~B}^{*}\right)\right)$ are dual to each other and, in particular, have the same dimension.

Indeed, these vector spaces now are finite-dimensional by the corollary to Theorem 4, and one applies Proposition 6 of [18].

REMARK. In Theorem 6 and its corollary, the mixed real-complex structure $F$ is reflected in the index pair $\left(n, n^{*}\right)$. This becomes perhaps somewhat more evident if we set $k=\operatorname{rank}(\mathbf{D}), g=\operatorname{rank}(\mathbf{G})$, so that $n=k+g$ and $n^{*}=g$. With this, formula (42) reads: 


$$
H^{q}\left(M, \mathbb{Q}^{p}(\mathrm{~B})\right)^{\prime} \cong H_{c}^{k+g-q}\left(M, \mathbb{Q}^{g-p}\left(\mathrm{~B}^{*}\right)\right) .
$$

Let, in particular, $p=0$, so that $\mathbb{Q}^{p}(\mathbf{B})=\mathbb{Q}^{0}(\mathbf{B})=\mathcal{S}_{\mathbf{F}}(\mathbf{B})$. Therefore, $H^{q}\left(M, \mathcal{S}_{\mathrm{F}}(\mathrm{B})\right)^{\prime}$ is isomorphic to $H_{c}^{n-q}\left(M, \mathbb{Q}^{g}\left(\mathrm{~B}^{*}\right)\right), n=\operatorname{rank}(\mathrm{F})$. This may be rewritten by introducing the canonical line-bundle $K_{M}=\bigwedge^{8} \overline{\mathbf{G}}^{*}$ of $(M, F)$ : indeed, $\mathbb{Q}^{g}\left(\mathbf{B}^{*}\right)$ is the sheaf of germs of $F$-holomorphic sections of $L_{a}^{g}\left(G, B^{*}\right)$ $\cong K_{M} \otimes \mathrm{B}^{*}$, so that we conclude that the dual of $H^{q}\left(M, \delta_{\mathrm{F}}(\mathrm{B})\right)$ is $H_{c}^{n-q}\left(M, \delta_{\mathrm{F}}\left(K_{M} \otimes \mathrm{B}^{*}\right)\right)$. A slightly different way of stating the same result is this: Since $K_{M} \otimes K_{M}^{*} \cong M \times \mathbf{C}, H^{q}\left(M, \delta_{\mathbf{F}}(\mathbf{B})\right)=H^{q}\left(M, \delta_{\mathbf{F}}\left(K_{M} \otimes K_{M}^{*} \otimes\right.\right.$ B)) $=H^{q}\left(M, \mathbb{Q}^{g}\left(K_{M}^{*} \otimes \mathrm{B}\right)\right)$ whose dual is $H_{c}^{n-q}\left(M, \delta_{\mathrm{F}}\left(K_{M} \otimes \mathrm{B}^{*}\right)\right)$ by (42).

As a special case, we also obtain, for $\mathbf{B}=M \times \mathbf{C}=\mathbf{B}^{*}$ and compact $M$,

$$
H^{q}\left(M, \mathcal{\Theta}_{\mathbf{F}}\right)^{*} \cong H^{n-q}\left(M, \delta_{\mathbf{F}}\left(K_{M}\right)\right),
$$

$n=\operatorname{rank}(\mathbf{F})$. Therefore, the arithmetical genus $\chi_{a}(M)$ coincides with $(-1)^{n} \chi_{F}\left(M, K_{M}\right)$ cf. e.g. [8].

One notes here that the canonical line-bundle $K_{M}$ also can be identified with $\Lambda^{g}\left(\overline{\mathbf{F}} / \mathbf{D}_{\mathbf{C}}\right)^{*}, g=\operatorname{rank}\left(\overline{\mathbf{F}} / \mathbf{D}_{\mathbf{C}}\right)$. In this form, no arbitrary splitting of $\mathbf{F}$ is involved: the canonical line-bundle depends only on $\mathbf{F}$.

\section{BIBLIOGRAPHY}

1. A. Borel and F. Hirzebruch, Characteristic classes and homogeneous spaces. I, Amer. J. Math. 80 (1958), 458-538; II, ibid. 81 (1959), 315-382.

2. N. Bourbaki, Variétés différentielles et analytiques, Hermann, Paris, 1971.

3. P. Dolbeault, Formes différentielles et cohomologie sur une variété analytique complexe. I, Ann. of Math. (2) 64 (1956), 83-130.

4. R. E. Edwards, Functional analysis, Holt, New York, 1965.

5. A. Frölicher, Relations between the cohomology groups of Dolbeault and topological invariants, Proc. Nat. Acad. Sci. U.S.A. 41 (1955), 641-644.

6. W. Greub, S. Halperin and R. Vanstone, Connections, curvature and cohomology. I, II, Academic Press, New York, 1972, 1973.

7. A. Grothendieck, Sur quelques points d'algèbre homologique, Tôhoku Math. J. 9 (1957), 119-221.

8. F. Hirzebruch, Topological methods in algebraic geometry, Springer-Verlag, New York 1966.

9. A. A. Kirillov, Elements of the theory of representations, Springer-Verlag, New York, 1976.

10. B. Kostant, MIT Seminar, 1967, also in: Lectures in modern analysis and applications. III, Lecture Notes in Math., vol. 170, Springer-Verlag, Berlin, 1970.

11. S. Lang, Introduction to differential manifolds, Addison-Wesley, Reading, Mass., 1972.

12. L. Nirenberg, A complex Frobenius theorem, Seminars on Analytic Functions. I, Princeton Univ. Press, Princeton, N. J., 1957.

13. R. Palais, Seminar on the Atiyah-Singer index theorem, Ann. of Math. Studies, Princeton Univ. Press, Princeton, N. J., 1965.

14. J. H. Rawnsley, On the cohomology groups of a polarization and diagonal quantisation, Trans. Amer. Math. Soc. 230 (1977), 235-255.

15. P. Renouard, Variétés symplectiques et quantification, thèse, Orsay, 1969.

16. G. de Rham, Variétés différentiables, Hermann, Paris, 1955.

17. K. S. Sarkaria, The de Rham cohomology of foliated manifolds, thesis, SUNY, Stony Brook, 1974. 
18. J.-P. Serre, Un théorème de dualité, Comment. Math. Helv. 29 (1955), 9-26.

19. F. Trèves, Topological vector spaces, distributions and kernels, Academic Press, New York, 1967.

20. I. Vaisman, Cohomolozy and differential forms, Dekker, New York, 1973.

21. A. Weil, Variétés kählériennes, Hermann, Paris, 1958.

22. R. O. Wells, Jr., Differential analysis on complex manifolds, Prentice-Hall, Englewood Cliffs, N. J., 1973.

Departmient of Mathematics, University of Massachusetts, Amaherst, Massachusetts 01003 